\title{
Deep brain stimulation in the central nucleus of the amygdala decreases 'wanting' and 'liking' of food rewards
}

\author{
Shani E. Ross, ${ }^{1}$ Emily Lehmann Levin, ${ }^{2}$ Christy A. Itoga ${ }^{3}$ Chelsea B. Schoen, ${ }^{4}$ Romeissa Selmane ${ }^{4,5}$ and \\ J. Wayne Aldridge $e^{2,4}$ \\ ${ }^{1}$ Biomedical Engineering Department, Ann Arbor, MI, USA \\ ${ }^{2}$ Department of Neurosurgery, Ann Arbor, MI, USA \\ ${ }^{3}$ Neuroscience Graduate Program, Ann Arbor, MI, USA \\ ${ }^{4}$ Department of Psychology, 530 Church Street, Ann Arbor, MI 48109-1043, USA \\ ${ }^{5}$ Department of Biology, University of Michigan, Ann Arbor, MI, USA
}

Keywords: food reward, neural recordings, operant responding, rat, taste reactivity

Edited by Rui Costa

Received 20 October 2015, revised 1 July 2016, accepted 4 July 2016

\begin{abstract}
We investigated the potential of deep brain stimulation (DBS) in the central nucleus of the amygdala (CeA) in rats to modulate functional reward mechanisms. The $\mathrm{CeA}$ is the major output of the amygdala with direct connections to the hypothalamus and gustatory brainstem, and indirect connections with the nucleus accumbens. Further, the CeA has been shown to be involved in learning, emotional integration, reward processing, and regulation of feeding. We hypothesized that DBS, which is used to treat movement disorders and other brain dysfunctions, might block reward motivation. In rats performing a lever-pressing task to obtain sugar pellet rewards, we stimulated the CeA and control structures, and compared stimulation parameters. During CeA stimulation, animals stopped working for rewards and rejected freely available rewards. Taste reactivity testing during DBS exposed aversive reactions to normally liked sucrose tastes and even more aversive taste reactions to normally disliked quinine tastes. Interestingly, given the opportunity, animals implanted in the CeA would self-stimulate with 500 ms trains of stimulation at the same frequency and current parameters as continuous stimulation that would stop reward acquisition. Neural recordings during DBS showed that CeA neurons were still active and uncovered inhibitory-excitatory patterns after each stimulus pulse indicating possible entrainment of the neural firing with DBS. In summary, DBS modulation of CeA may effectively usurp normal neural activity patterns to create an 'information lesion' that not only decreased motivational 'wanting' of food rewards, but also blocked 'liking' of rewards.
\end{abstract}

\section{Introduction}

Could deep brain stimulation (DBS) treat addiction (Rouaud et al., 2010; Heldmann et al., 2012) or obesity (Sani et al., 2007; Melega et al., 2012)? Here, we assess the central nucleus of the amygdala (CeA) as a potential target for neuromodulation of reward dysfunction to mimic DBS treatments for Parkinson's disease (Schwalb \& Hamani, 2008; Gubellini et al., 2009; Weaver et al., 2009; Doshi, 2011) and other disorders (Chang, 2004; Gubellini et al., 2009) including OCD (Haber \& Brucker, 2009; Greenberg et al., 2010), depression (Mayberg et al., 2005; Lozano et al., 2008), and epilepsy (Halpern et al., 2008).

DBS mechanisms remain an enigma although it is thought to be due to cell inhibition and axon activation (Garcia et al., 2005a; Kringelbach et al., 2007; Okun, 2012). Modeling suggests that

Correspondence:

Dr J. Wayne Aldridge, ${ }^{4}$ Department of Psychology, as above.

E-mail: jwaynea@umich.edu high-frequency stimulation $(>100 \mathrm{~Hz}$ ) may impose a regular pattern in neural circuits effectively 'masking' pathological activity patterns (Grill et al., 2004; Johnson \& McIntyre, 2008). Neural recording during stimulation could provide useful insight into DBS mechanisms.

Here, we targeted the central nucleus of the amygdala (CeA), which has connections to cortical and subcortical areas involved in processing taste, food consumption, and reward including the lateral hypothalamus, ventral tegmental area (Sah et al., 2003), parabrachial nucleus and nucleus of the solitary tract (Ricardo \& Koh, 1978) and indirect connections with the nucleus accumbens and ventral pallidum (Price \& Amaral, 1981). The CeA is composed largely of medium-sized spiny neurons, like the ventral striatum (McDonald \& Augustine, 1993) and its afferents and efferents are primarily GABA-ergic (Swanson \& Petrovich, 1998). The CeA is involved in reward learning, reward processing, and regulation of feeding (Baxter \& Murray, 2002), although its specific role is still debated (Cai et al., 2014). Incentive motivational mechanisms may play a role as 
decreased food consumption occurs with CeA lesions and inactivation (Kemble et al., 1979; Seeley et al., 1993; Touzani et al., 1997; Will et al., 2004, 2009) along with increased food intake with opioid stimulation (Mahler \& Berridge, 2009; DiFeliceantonio \& Berridge, 2012). Furthermore, CeA activation may focus and intensify incentive motivation (Robinson et al., 2014). The hedonic functions of $\mathrm{CeA}$ are less clear with reports of both increased aversive reactions to bitter tastes with CeA lesions (Touzani et al., 1997), as well as reports of unchanged 'liking' or 'disliking' reactions (Kemble et al., 1979; Galaverna et al., 1993; Mahler \& Berridge, 2011) although incentive control by DBS would still be fortuitous.

In this study, we stimulated CeA with DBS-like parameters hypothesizing and demonstrating that we could modulate (i) working for, (ii) approach and (iii) consumption of food rewards. Specifically, we showed that lever pressing for sucrose pellet rewards was reduced by DBS in CeA. The effect was not due to motor disruption, but could be due in part to observed changes in hedonic evaluation of tastes. CeA stimulation made taste stimuli aversive in general. Simultaneous neural recordings during DBS showed fewer neural responses to reward-related behavior.

\section{Materials and methods}

Male Sprague-Dawley rats, $\sim 8-13$ weeks old (250-400 g, Charles River, US) were used as subjects $(n=20$ total, 11 in experiment 1 , 9 in experiment 2). Rats were housed individually on a 9:30 a.m. to 7:30 p.m. reversed light/dark schedule with unrestricted access to standard rat chow and water (animals were paired-housed prior to electrode implantation). Testing was carried out during their dark (active) period. Two experiments were performed to investigate the effects of deep brain stimulation (DBS) in the central nucleus of the amygdala (CeA) on reward behavior. Experiment 1 assessed the effects of DBS on an operant responding task. Experiment 2 assessed the effects of DBS on 'liking' and 'disliking' reactions to tastes. All procedures were approved by the University of Michigan Institutional Animal Care and Use Committee, in accordance with the National Institute of Health's guidelines for the care and use of laboratory animals.

\section{Experiment 1 (operant task)}

\section{Apparatus}

All training and testing were conducted in a $28 \mathrm{~cm}$ $\times 35 \mathrm{~cm} \times 60 \mathrm{~cm}$ plastic chamber illuminated by red light from below with a metal grid (to ground the animal) over a glass floor. The rat's electrode was connected to the recording and stimulating system via a headstage cable and commutator. The commutator enabled the rat to freely move around and explore the chamber. For the operant responding task, two retractable levers (Coulbourn Instruments, Whitehall, PA, US) were attached to one wall of the chamber and located on either side of a food bowl. Touch detectors were connected to both levers in order to assess lever contacts. One of the levers was designated as the reward lever and delivered a sucrose pellet to the food bowl contingent upon lever contacts. The other lever served as a control for general lever-pressing behavior, where responses were recorded, but had no consequences. The location of the reward and control lever was counter-balanced across rats and remained consistent for each rat throughout the study.

In a separate self-stimulation study, levers were retracted and two 2.5-cm-diameter nose-poke holes with photo-beam detectors were exposed on the cage wall. Poking in a designated reward hole (counter-balanced between animals), triggered intracranial stimulation. Poking the control hole did nothing (timing was recorded).

Delivery of all stimulus and reward presentations, as well as recording of responses were managed by MTASK (in-house program). Electrical stimulation was delivered using a constant-current digital stimulus isolator (A-M systems, Carlsborg, WA, US) with timing (frequency and pulse width) controlled by a LabVIEW (National Instruments, Austin, TX, US) program developed in the lab. Neural activity was recorded during the test sessions using an in-house program, DataTask (written in LabVIEW). Neural signals were amplified $(\times 1000)$ and bandpass-filtered between $300 \mathrm{~Hz}$ and $6 \mathrm{kHz}$. This relatively low gain was used to prevent amplifier saturation and consequent lock-out by the large amplitude stimulation artifacts. A video camera underneath the glass floor recorded all behavior. Timestamp clocks (10 $\mu$ s accuracy) for the behavior control program, video recording, and neural recording were synchronized to enable subsequent analysis of neural activity related to stimulus presentations and behavioral responses.

\section{Behavioral training}

Habituation. Three to five days prior to the start of training, rats were handled for 10-15 min daily and given free access to bananaflavored sucrose pellets (used throughout experiment 1) in their home cages (10-15 pellets per day). One day prior to training, rats were placed in the chamber for $30 \mathrm{~min}$ with the levers retracted to acclimatize them to the testing chamber.

Food self-administration paradigm. Ten minutes after an animal was placed in the chamber, both levers extended into the chamber where they remained for the rest of the 40-min session. Training began with a fixed ratio schedule one (FR1) in which each contact of the reward lever delivered one sucrose pellet, followed by a 500$\mathrm{ms}$ timeout. When the rats delivered more than 10 pellets and touched the reward lever three times more than the control lever, they were then trained on a limited progressive ratio paradigm (5 FR1 trials, 5 FR5 trials, and then FR10 trials for the remainder of the session). The successful delivery of five pellets triggered the shift from FR1 to FR5 and again from FR5 to FR10. There was a 500-ms timeout period between lever contacts (Fig. 1a.i). Once animals had consistent average lever responses across three consecutive days, they were implanted with electrodes.

\section{Surgical procedures}

Electrodes consisted of two moveable bundles of six wires (two $75-\mu \mathrm{m}$ stainless steel stimulating wires, four $50-\mu \mathrm{m}$ tungsten recording wires). Each bundle could be lowered independently. Under 1-2\% isoflurane anesthesia with oxygen maintenance $(1-1.5 \mathrm{~L} / \mathrm{min})$, electrodes were fixed to the skull stereotaxically (Tindell et al., 2004) targeting the central nucleus of the amygdala (AP $-2.0 \mathrm{~mm}, \mathrm{ML} \pm 3.4 \mathrm{~mm}$ to $\pm 3.5 \mathrm{~mm}$, DV $7.0 \mathrm{~mm}$ to $8.5 \mathrm{~mm}$ ) in seven rats. Four surgical/stimulation control rats had electrodes in the ventral pallidum, extended amygdala, medial anterior dorsal nucleus of the amygdala, amygdala striatum transitional area, and inferior to the CeA. Thermal support was provided and vital signs were monitored during the procedure. For pain management, animals were given Flunixin meglumine $(2.5 \mathrm{mg} / \mathrm{kg}$ subcutaneously) prior to removal from anesthesia and also twice the next day (and subsequent days if needed). A thin layer of lidocaine jelly and bacitracin was placed around the wound edge after closure and the animal was given $1-2 \mathrm{~mL}$ of warm sterile saline $(0.9 \%)$ intraperitoneally to help prevent dehydration. After anesthesia recovery, animals were 
i Session overview

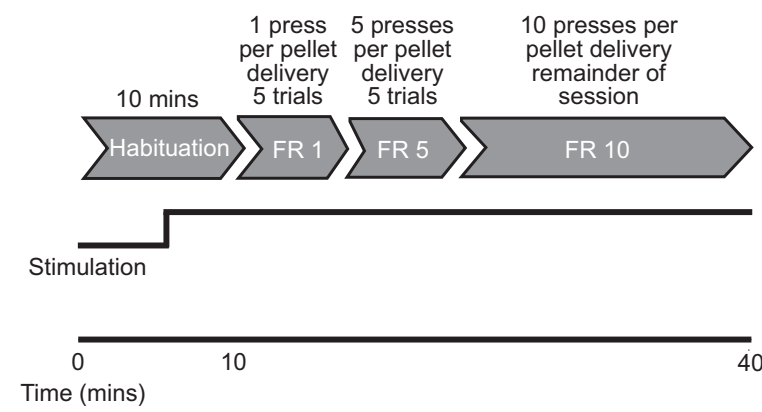

\section{ii Experimental timeline}

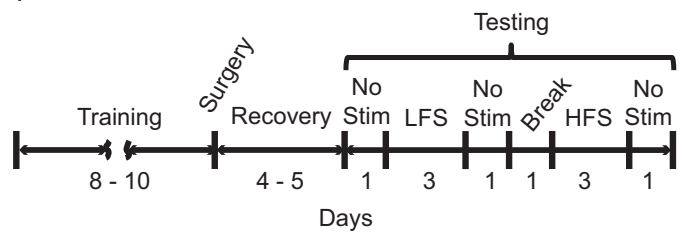

b

Experiment 2 task design

i Session overview

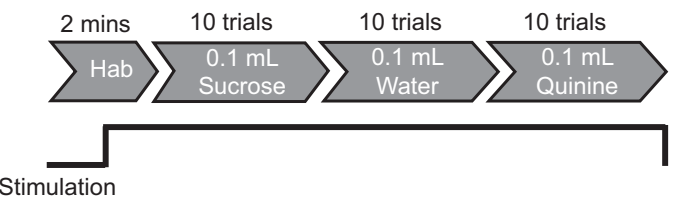

ii Taste infusion trial

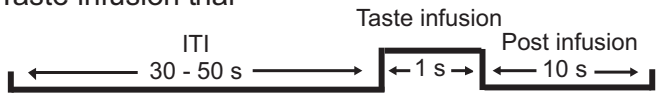

iii Experimental timeline

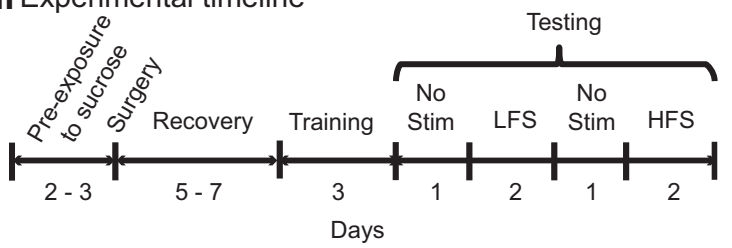

FIG. 1. (a) Experiment 1 task design. (i) Schematic of a limited progressive ratio (FR - fixed ratio) test session. Stimulation was turned on 5 min into the habituation period for DBS test days. (ii) Experimental timeline. (b) Experiment 2 task design. (i) Experimental paradigm for a given test session. For DBS test sessions, stimulation was turned on $1 \mathrm{~min}$ into the habituation period. (ii) Schematic of a taste-infusion trial showing timing of the different events (ITI - intertrial interval). (iii) Experimental timeline. No Stim - test days where no stimulation was delivered, LFS - low frequency stimulation at $20 \mathrm{~Hz}$, HFS - high-frequency stimulation at $130 \mathrm{~Hz}$.

housed individually with free access to food and water. Penicillin G benzathine (15 000 units/daily subcutaneously) was given immediately after surgery and the following day. Rats were given at least 4 days to recover before screening tests.

\section{Screening}

Screening tests were used to search for the optimal electrode depth location and lowest effective current. Each screening session consisted of a 1-min habitation period and 10-min operant response paradigm (described in Behavioral training). Stimulation was delivered continuously at $130 \mathrm{~Hz}$ (see Stimulation parameters). If stimulation had no effect on pellet delivery or produced motor side effects (e.g. paw twitching, turning), both electrodes were lowered in increments of $0.16 \mathrm{~mm}$. Electrode advancement stopped when stimulation caused a cessation of pellet delivery and there were no adverse motor effects. Searching was also stopped at $8.5 \mathrm{~mm}$ below dura if no site was found. Stimulation current started at $250 \mu \mathrm{A}$ but was increased up to $400 \mu \mathrm{A}$, if no effect was observed, or decreased to a lower current. Four screening tests were done per day with stimulation absent for one of the sessions (to provide a daily baseline response). Once the effective site and stimulation amplitude were found, rats were given 2 days off and then moved on to the limited progressive ratio test described below.

\section{Testing DBS-like stimulation}

The effect of stimulation on a limited progressive ratio operant responding task (described above under Food self-administration paradigm) was tested in 40-min sessions, one per day (Fig. 1a.i). A testing sequence consisted of three consecutive days of high-frequency stimulation $(130 \mathrm{~Hz})$, three consecutive days of low frequency stimulation $(20 \mathrm{~Hz})$, and 3 days without stimulation (Fig. 1a.ii). A two-day break was given between high and low frequency days to minimize any potential lingering effects of DBS
(Gubellini et al., 2009). Rats were randomly selected to begin testing with either the high or low frequency stimulation block and no stimulation days were interspersed throughout the 2-week testing period. On stimulation days, stimulation was turned on 5 minutes into the habituation period to allow animals to acclimatize to the stimulation and to provide a period of assessment with stimulation before the behavioral task began. In some cases, extra test days were used to record more neural data.

In order to determine the threshold frequency that resulted in decreased operant responses, additional tests were run on two of the rats implanted in the CeA. These two rats were given a break of several days after the main experiment, then they were tested on the same behavioral task as described above but with lower frequencies of stimulation: $2 \mathrm{~Hz}, 4 \mathrm{~Hz}, 10 \mathrm{~Hz}$, and $16 \mathrm{~Hz}$. Each frequency was tested (on a separate day) and repeated and the number of lever contacts and pellets consumed were measured. Rats were also retested with $20 \mathrm{~Hz}$ stimulation to verify that stimulation at this frequency still resulted in decreased operant responses and that the effects of stimulation had not changed over time. They were also tested again in the absence of stimulation.

\section{Intracranial self-stimulation}

A few days after the food self-administration test, rats were trained to poke their nose in a hole to receive a $500 \mathrm{~ms}, 130 \mathrm{~Hz}$ stimulation train (500 ms timeout period between pokes). Self-stimulation was acquired when the subject poked his nose into the reward hole three times more than the control hole for two consecutive days (note, three of the rats had been trained at $100 \mathrm{~Hz}$ and their self-stimulation training and testing days were done before FR testing). To determine the frequency of stimulation that best supports self-stimulation, subjects were then tested on frequency sweeps. Frequencies of $20 \mathrm{~Hz}, 60 \mathrm{~Hz}$, and $130 \mathrm{~Hz}$ were tested each for $15 \mathrm{~min}$ and the numbers of nosepokes for the reward and control holes were quantified for each frequency. Order of frequency testing was randomized. 


\section{Stimulation parameters}

Stimulation values were controlled by an A-M systems stimulator and were set to continuous monopolar, biphasic stimulation with pulse width of $100 \mu$ s per phase and amplitude 250-400 $\mu$ A. Stimulation was split between two identical bilateral electrodes each $75 \mu \mathrm{m}$ in diameter so current delivered to the target on each side of the brain was $\sim$ half of the set amplitude, that is, around $125 \mu \mathrm{A}$ to $200 \mu \mathrm{A}$. Each rat was tested at its minimum threshold current determined in the screening test. Pilot current studies (starting from $20 \mu \mathrm{A}$ ) had also been performed which also gave a starting amplitude range (see Supporting information, Data S1 and Fig. S1). The majority of the rats had a minimum effective current of $250 \mu \mathrm{A}$ (or $125 \mu \mathrm{A}$ per electrode/location) resulting in a current density of $\sim 28.3 \mathrm{~mA} / \mathrm{mm}^{2}$ at the electrode tip (see Supporting information for current density calculations). At a distance of $\sim 0.2 \mathrm{~mm}$ and $\sim 0.5 \mathrm{~mm}$ from the tip, this current density would approximate the threshold to activate cell bodies and axons of passage (Ranck, 1975; Nowak \& Bullier, 1998) with a volume of activation of up to $\sim 0.5 \mathrm{~mm}^{3}$ (see also Data S1).

Given the frequency dependencies in clinical DBS applications, we wanted to test this out as well in our study. Hence, we chose to assess two 'extreme' frequencies - a high and low frequency. We selected a standard high frequency $(130 \mathrm{~Hz})$ shown to be therapeutic in clinical DBS (Okun, 2012) and a low frequency $(20 \mathrm{~Hz})$ shown to have either no effect or an activating effect (Kuncel et al., 2007).

\section{Histology}

Anatomical localization of electrode sites was done after completion of testing. Rats were anesthetized with isoflurane gas. A lesioning DC current $(0.1 \mathrm{~mA})$ was passed for $10 \mathrm{~s}$ to mark the electrode location in the brain. After euthanizing the rats with pentobarbital (dose: $200 \mathrm{mg} / \mathrm{kg}$, intraperitoneal), the brains were removed, frozen in an isopentane and isopropyl alcohol solution, sliced into $40 \mu \mathrm{m}$ sagittal sections using a CM 1850 cryostat (Leica Microsystems, Buffalo Grove, IL, US), and stained with cresyl violet (Fisher Scientific, Pittsburgh, PA, US). Electrode placement was confirmed by examining the lesion sites in stained slices under a light microscope (Nikon, Melville, NY, US).

\section{Behavioral assessment and data analysis}

To determine the effects of stimulation on motivation, lever contacts, pellets consumed and approaches to the food bowl were counted. Lever contacts on the control and reward levers were compared over each 30-min testing session. Pellet consumption was calculated (delivered minus unconsumed pellets).

Motor and exploratory behavior and approaches to the food bowl were assessed by frame-by-frame video analysis with DataRat (inhouse video scoring software) during the last $5 \mathrm{~min}$ of the habituation period and for 30 -s intervals every 5 min during operant testing. Horizontal and vertical midline chamber crossings, rearing movements, and 'vacuum' taste reactions (Berridge \& Valenstein, 1991) were counted.

\section{Neural analysis}

Single neural units were discriminated from each other and background noise (Tindell et al., 2004) using Offline Sorter (Plexon, Inc., Dallas, TX, US). Digitized spikes in the database were analyzed with Epoch Builder (in-house neural and behavioral analysis program) and Neuroexplorer (Nex Technologies, Madison, AL, US). The effects of stimulation on neural activity were assessed after stimulation artifacts were removed. Firing rate calculations during stimulation periods were corrected for loss of neural data due to the artifact dead time (Bar-Gad et al., 2004). Stimulation artifacts were removed using a custom-built LabVIEW program that fitted each artifact independently with a 7 th degree polynomial and subtracted the fit from the recording (using an approach based on Wagenaar \& Potter (2002)).

The immediate effects of stimulation were determined by peristimulus time histograms (PSTHs, bin width $=0.5 \mathrm{~ms}$ ) aligned to the onset of each stimulation pulse (for all stimulation pulses in the session). Increases or decreases in firing rate were deemed significant if two consecutive bins were greater than \pm 2 standard deviations from the mean firing rate of the unit measured during the first $5 \mathrm{~min}$ of habituation (before stimulation). Control 'PSTH' plots were computed in an identical manner from the 5-min prestimulation period by aligning to 'sham stimulation' pulses simulating $20 \mathrm{~Hz}$ or $130 \mathrm{~Hz}$ stimulation.

Perievent time histograms (PETHs) and rasters were analyzed for reward-related events: pellet delivery, first lever contact (in each trial) on the reward lever, and on the control lever. The firing rate reference for all unit responses was the average rate during the $5 \mathrm{~s}$ just before the habituation period ended. To determine whether a unit was responsive to pellet delivery, firing rates during the $0.5-\mathrm{s}$ epoch after the stimulus event was compared to the reference period. Neural activity before and following lever response was also evaluated by comparing the 0.5 -s period just before and after the first lever contact to the 5-s reference period.

\section{Experiment 2 (taste reactivity)}

All training and testing were conducted with rats in a $25-\mathrm{cm}$ diameter plastic cylinder over a clear floor in white light for video recording from below (Tindell et al., 2006). Taste reactions (Grill \& Norgren, 1978) were quantified from a frame-by-frame analysis of video recordings.

\section{Habituation and exposure to sucrose solution}

Prior to surgery, rats were handled for $10-15 \mathrm{~min}$ and then given daily access to $20-\mathrm{mL}, 17 \%$ sucrose solution in their home cage for 4 days. Rats that did not consume at least $15 \mathrm{~mL}$ of sucrose solution per day by day 4 were excluded from the study. For 2 days before surgery, rats were placed in the chamber for $10 \mathrm{~min}$ to acclimatize to the experimental set-up. Rats then underwent oral cannula and electrode implantation surgery as described below.

\section{Surgical procedures}

Bilateral oral cannula implantation was performed as described in a previous study (Tindell et al., 2006). In the same surgery, rats were then implanted with a recording and stimulating electrode targeting the central nucleus of the amygdala (AP $-2 \mathrm{~mm}, \mathrm{ML} \pm 3.4 \mathrm{~mm}$ to $\pm 3.5 \mathrm{~mm}$, DV $7.5-8.5 \mathrm{~mm}$ ) on each side of the brain. Electrode construction, electrode implantation procedure and peri-operative care were the same as described in experiment 1 with the addition that penicillin was given pre-operatively as well as daily for up to 5 days after surgery.

\section{Taste reactivity training}

After surgical recovery (5-7 days), rats underwent 3 days of tasteinfusion training in the testing chamber. Sessions consisted of a 2- 
min habituation period, followed by trials of intraoral infusions of sucrose, water, and quinine with a variable interval of $40 \mathrm{~s}$ to $60 \mathrm{~s}$ between trials. For each session, rats were given blocks of 10 trials per taste, starting with $17 \%$ sucrose solution, followed by tap water, and then $0.01 \%$ quinine (Fig. 1b.i). Between blocks, an extra infusion of tap water was given in order to rinse the mouth between tastes. For each trial, $0.1 \mathrm{~mL}$ of the tastant was infused over a period of $1 \mathrm{~s}$ directly into the rat's mouth using a similar set-up as described by Tindell et al. (2006) (Fig. 1b.ii). After taste reactivity training but before testing, rats were also given screening tests to determine effective electrode depth and minimum effective current that produced mouth movements. Screening tests proceeded similar to those done in experiment 1 (see Screening), although instead of doing an operant response task, the animals' facial reactions were observed. After screening, rats received 2 days off before taste reactivity testing began.

\section{Taste reactivity testing with $D B S$}

Rats were tested over a period of 6 days. Video recordings of taste reactions were made with the camera zoomed in and focused on the mouth and forelimb region. Testing conditions were similar to training conditions, although rats received stimulation during testing on four of the 6 days and neural activity was recorded. For three of the nine rats used in experiment 2 , stimulation was delivered using a Medtronic pulse generator (Minneapolis, MN, US) (3-5 V or $\sim 72$ to $\sim 150 \mu \mathrm{A}$, monophasic $100 \mu \mathrm{s}$ pulses). Stimulation frequencies tested were the same as in experiment 1 . For all other rats, the same stimulation parameters as in experiment 1 were used. For DBS sessions, stimulation was turned on one minute into the habitation period and remained on until the end of the session. Rats were tested first with a no stimulation session, followed by 2 days of either high or low frequency, then another day of no stimulation, followed by 2 more days of the other frequency of stimulation (Fig. 1b.iii: experimental timeline). The order of stimulation was counter-balanced across rats. Anatomical localization of electrode sites was done after completion of testing as described in experiment 1 .

\section{Behavioral assessment and data analysis}

Taste reactions (Grill \& Norgren, 1978) were scored offline using frame-by-frame video analysis and an in-house DataRat scoring program. For each taste, five out of ten trials were analyzed. Hedonic, neutral, and aversive taste reactions were scored and summed (Berridge, 2000) during the 10 -s period beginning at the onset of the infusion. To assess if stimulation alone produced any 'vacuum' facial and body reactions (specifically mouth, tongue, or forelimb movements) without any actual taste present (Berridge \& Valenstein, 1991), two $10 \mathrm{~s}$ periods (immediately before taste infusion and $30 \mathrm{~s}$ before infusion) were also scored for reactions. These two periods were later combined when statistical testing revealed no significant difference between them.

\section{Neural analysis}

Discrimination and stimulation artifact removal with dead time compensation was the same as experiment 1 . Baseline firing rates were computed in the habituation period before and after stimulation started. As in experiment 1, PSTHs and PETHs were computed. PETHs were analyzed around taste onset. To determine whether a unit was responsive to taste onset, firing rates during $1 \mathrm{~s}$ epochs after the stimulus event were compared to the $1 \mathrm{~s}$ period before the event.

\section{Statistical analysis (experiment 1 and 2)}

Numbers are reported as mean \pm standard error. Unless otherwise stated two-way mixed models ANOvas were used to test the main effects of stimulus location (between-subjects) and frequency of stimulation (within-subjects, repeated measure) for the different behavioral measures in experiment 1 . The differences between reward and control lever contacts for each test condition were assessed to determine overall effects of frequency and/or structure being targeted. Paired t-tests were also carried out to compare differences between reward and control lever contacts for a given frequency and between reward and control nose-pokes for self-stimulation results. For experiment 2, three-way mixed models ANOVAS were carried out comparing taste reactions (hedonic, neutral, and aversive), frequency of stimulation, and period (before and during taste infusion) for a given taste. For each taste reaction, the tastes, frequency of the stimulation, and period were compared. For both experiments, Mann-Whitney U tests were used to determine if a response was significant to a given event for individual units. Also, given the limited number of units to begin with, all units in a given test condition were pooled across rats. Two-way ANOvas were carried out comparing different epochs and stimulation conditions $(0 \mathrm{~Hz}, 20 \mathrm{~Hz}$, and $130 \mathrm{~Hz}$ ) for a given event of interest. Statistical tests were implemented with SPSS vs. 22 (IBM, Armonk, NY, US). Alpha was set to 0.05 and specific comparisons were made using Holm-Sidak post hoc tests when significant effects were found. The standardized effect size is also reported for major comparisons. For a mixed models ANOVA, the standardized effect size (ES) was computed from Eqn (1) in Mathematics and Equations (Spybrook et al., 2011).

$$
\begin{aligned}
\text { ES } & =\frac{\hat{\beta}}{\sqrt{\hat{\tau}^{2}+\hat{\sigma}^{2}}}, \text { where } \hat{\beta}=\text { fixed coefficient, } \hat{\tau}^{2} \\
& =\text { variance of the intercept, and } \hat{\sigma}^{2}=\text { covariance }
\end{aligned}
$$

\section{Results \\ Experiment 1 (operant responding task) \\ $D B S$ in the central nucleus of the amygdala (CeA) decreased working for sucrose pellets}

Stimulation of CeA ( $n=7$ rats, Fig. 2a) significantly decreased engagement with the reward lever (Fig. 3a and b) compared to no stimulation and to stimulation in control sites $(n=4$ rats, Fig. 2a) [stimulation site $\mathrm{x}$ frequency: $\left.F_{2,102}=22.47, P<0.001\right]$. Screening for the effective sites (Fig. 2) indicated that the volume of tissue activated by stimulation was about $0.4 \mathrm{~mm}^{3}$ (Fig. S1). Both $20 \mathrm{~Hz}$ and $130 \mathrm{~Hz}$ decreased reward lever contacts, relative to control lever contacts, when compared to no stimulation $(P<0.001, \mathrm{ES}=2.95$ and $P<0.001$, $\mathrm{ES}=2.10$ respectively). There was no difference between high and low frequency $(P=0.08, \mathrm{ES}=0.885)$. For rats implanted in the control sites, there was no effect on lever contacts of either $20-\mathrm{Hz}$ stimulation or $130-\mathrm{Hz}$ stimulation $(P=0.94, \mathrm{ES}=0.22$ and $P=0.95$, $\mathrm{ES}=0.03$ respectively) and no difference between frequencies $(P=0.94, \mathrm{ES}=0.25)$. With stimulation off, the number of reward lever contacts (relative to control lever contacts) was similar for electrodes in CeA and non-CeA (control) sites $(P=0.24, \mathrm{ES}=0.15)$.

\section{DBS in the CeA decreases consumption of sucrose pellets}

Pellet delivery and consumption. Stimulation of the CeA also resulted in dramatically fewer lever presses and thus, decreased sugar pellet delivery and consumption (Fig. 3c) [stimulation site $\mathrm{x}$ frequency: $\left.F_{2,105}=19.941, P<0.001\right]$. DBS in CeA diminished 
a Experiment 1 histology results

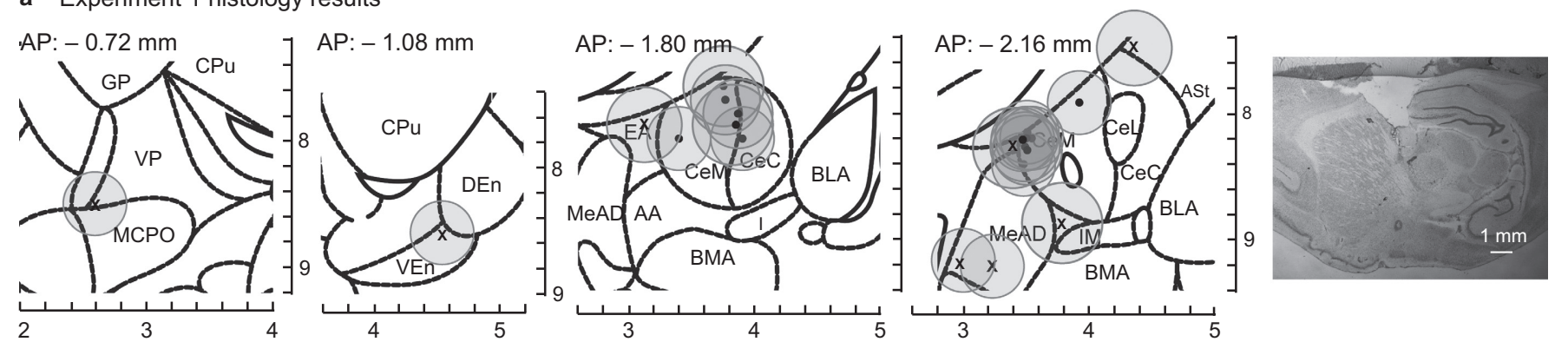

b Experiment 2 histology results
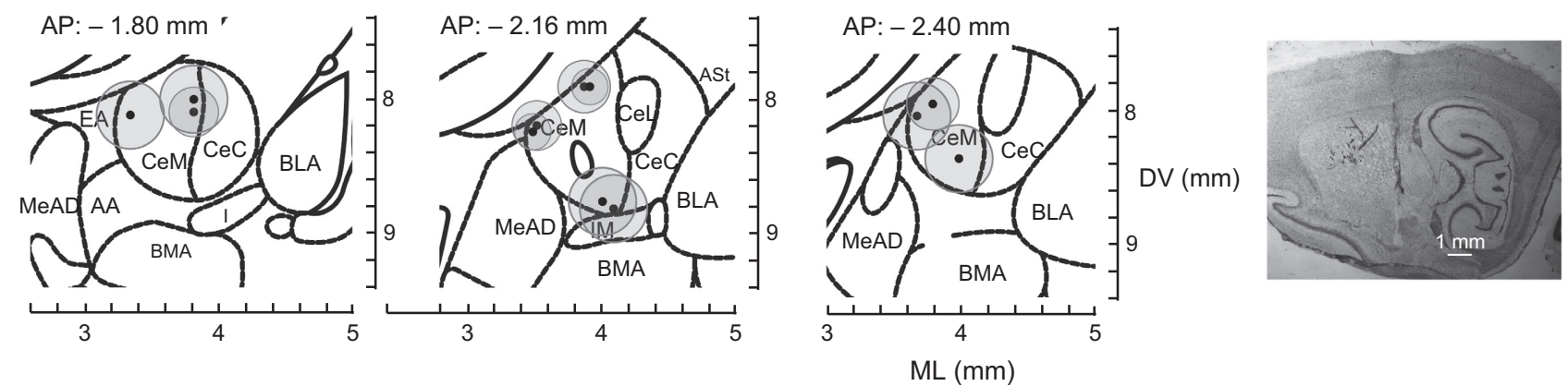

FIG. 2. Coronal slices showing electrode placements of rats implanted in the CeA (black-filled circles, $n=7$ rats) and in the controls sites (x's, $n=4$ rats) for (a) experiment 1 and (b) experiment $2(n=6$ rats). Gray circles represent estimated current density that would exceed the threshold to activate axons (for cell bodies, circles would be $\sim 1 / 2$ the size of these). See Data S1 for detailed information. Note, rats were implanted bilaterally, but for presentation purposes both left and right electrode locations are plotted on the same coronal image in the figure. Images are modified from the Paxinos and Watson rat atlas, 6th edition (Paxinos \& Watson, 2007). See Abbreviations section, for names of the structures. Images on the far right are examples of brain slices showing electrode tracks and lesion marks from a rat implanted in the $\mathrm{CeA}$ in experiment 1 (top, far right) and another in experiment 2 (bottom, far right). Slices stained with cresyl violet.

pellet consumption to less than one-sixth of that was eaten when stimulation was absent ( 0 vs. $20 \mathrm{~Hz}: P<0.001$, ES $=3.87 ; 0$ vs. $130 \mathrm{~Hz}: \quad P<0.001$, ES $=3.43)$, but there was no difference between frequencies $(P=0.29, \mathrm{ES}=0.44)$. In contrast, stimulation in control sites had no effect on pellet consumption ( 0 vs. $20 \mathrm{~Hz}$ : $P=0.16, \mathrm{ES}=1.09 ; 0$ vs. $130 \mathrm{~Hz}: P=0.92, \mathrm{ES}=0.07)$ and no difference between frequencies $(P=0.11, \mathrm{ES}=1.16)$ (Fig. 3c). Baseline consumption was the same between rats implanted in the $\mathrm{CeA}$ and in control sites $(P=0.20, \mathrm{ES}=0.87)$.

During CeA stimulation, even the few pellets earned were rarely eaten, and the occasional pellets that rats did put in their mouths were often expelled (Movie S1) [stimulation site $\times$ frequency: $\left.F_{2,105}=12.60, P<0.001\right]$. On average, rats ate only $21.9 \pm 12.1 \%$ of the pellets delivered during $20 \mathrm{~Hz}$ stimulation into the $\mathrm{CeA}$ and $57.5 \pm 19.3 \%$ during $130 \mathrm{~Hz}$ stimulation compared to $99.8 \pm 0.1 \%$ during no stimulation ( 0 vs. $20 \mathrm{~Hz}: P<0.001$, $\mathrm{ES}=1.85$; 0 vs. $130 \mathrm{~Hz}: P=0.02, \mathrm{ES}=1.01)$. There was no difference between $20 \mathrm{~Hz}$ and $130 \mathrm{~Hz}$ stimulation $(P=0.06, \mathrm{ES}=0.84)$. Rats implanted in control sites ate almost $100 \%$ of pellets delivered whether receiving stimulation or not $(0 \mathrm{~Hz}: 99.4 \pm 0.2 \%, 20 \mathrm{~Hz}: 99.8 \pm 0.2 \%$, $130 \mathrm{~Hz}: 99.5 \pm 0.3 \% ; P=1, \mathrm{ES} \leq 0.01$ for all comparisons).

Approach. The approach rate after pellet delivery was compared as an index of motivation. Rats typically approached the food bowl immediately after lever pressing to retrieve the pellets. However, with CeA stimulation at both $20 \mathrm{~Hz}$ and $130 \mathrm{~Hz}$, rats approached the food bowl less frequently than when stimulation was absent $(P<0.001$, $\mathrm{ES}=4.57$ and $P<0.001, \mathrm{ES}=3.70$ respectively, Fig. $3 \mathrm{~d})$. There was a significant interaction effect between stimulation site and frequency $\left[F_{2,103}=24.48, P<0.001\right]$. Stimulation at control sites had no effect on approach behavior $(P=0.059, \quad \mathrm{ES}=1.09$ and
$P=0.745, \mathrm{ES}=0.09$ for $20 \mathrm{~Hz}$ and $130 \mathrm{~Hz}$ respectively). With stimulation absent, there was no difference in approaches to the bowl between $\mathrm{CeA}$ and non-CeA rats $(P=0.29, \mathrm{ES}=0.77)$.

'Vacuum' taste reactions. Stimulation in the CeA appeared to induce a state of taste aversion (see experiment 2) as shown by mouth gaping movements. Twenty-Hertz stimulation in the CeA produced on average $22.9 \pm 12.5$ gapes per 30-min test session. With $130-\mathrm{Hz}$ stimulation, this number was $31.2 \pm 8.0$ gapes in 30 min. No gapes were observed during sessions with no stimulation or for control sites.

\section{General exploratory behavior}

Chamber crossing and rearing. Stimulation had little or no effect on general motor behavior (assessed during the last $5 \mathrm{~min}$ of the habituation period). Chamber crossing did not change significantly with $\mathrm{CeA}$ stimulation [main effect: stimulation site $F_{1,105}=0.58, P=0.45$, $\mathrm{ES}=0.17 ;$ main effect: frequency $F_{1,105}=4.68, \quad P=0.03$, $\mathrm{ES}=0.26$; stimulation site $\mathrm{x}$ frequency $\left.F_{1,105}=2.69, P=0.10\right]$. Average rate of chamber crossing for $\mathrm{CeA}$ rats was $6.45 \pm 0.62 \mathrm{crosses} / \mathrm{min}$ during no stimulation and $3.20 \pm 0.82$ crosses/min with stimulation. Rats implanted in control sites did $4.16 \pm 0.12 \mathrm{crosses} / \mathrm{min}$ and $3.86 \pm 0.30 \mathrm{crosses} / \mathrm{min}$ during no stimulation and stimulation respectively. Measures of rearing behavior also confirmed the absence of stimulation effects on general movement [main effect: stimulation site $F_{1,105}=1.02, P=0.32, \mathrm{ES}=0.23$; main effect: frequency $F_{1,105}=2.89, P=0.09$, ES $=0.30$; stimulation site x frequency $\left.F_{1,105}=1.42, P=0.24\right]$. Average rears per minute were $3.44 \pm 0.60$ (no stimulation) and $1.78 \pm 0.37$ (during stimulation) for rats implanted in the CeA. Similarly, the average rears 

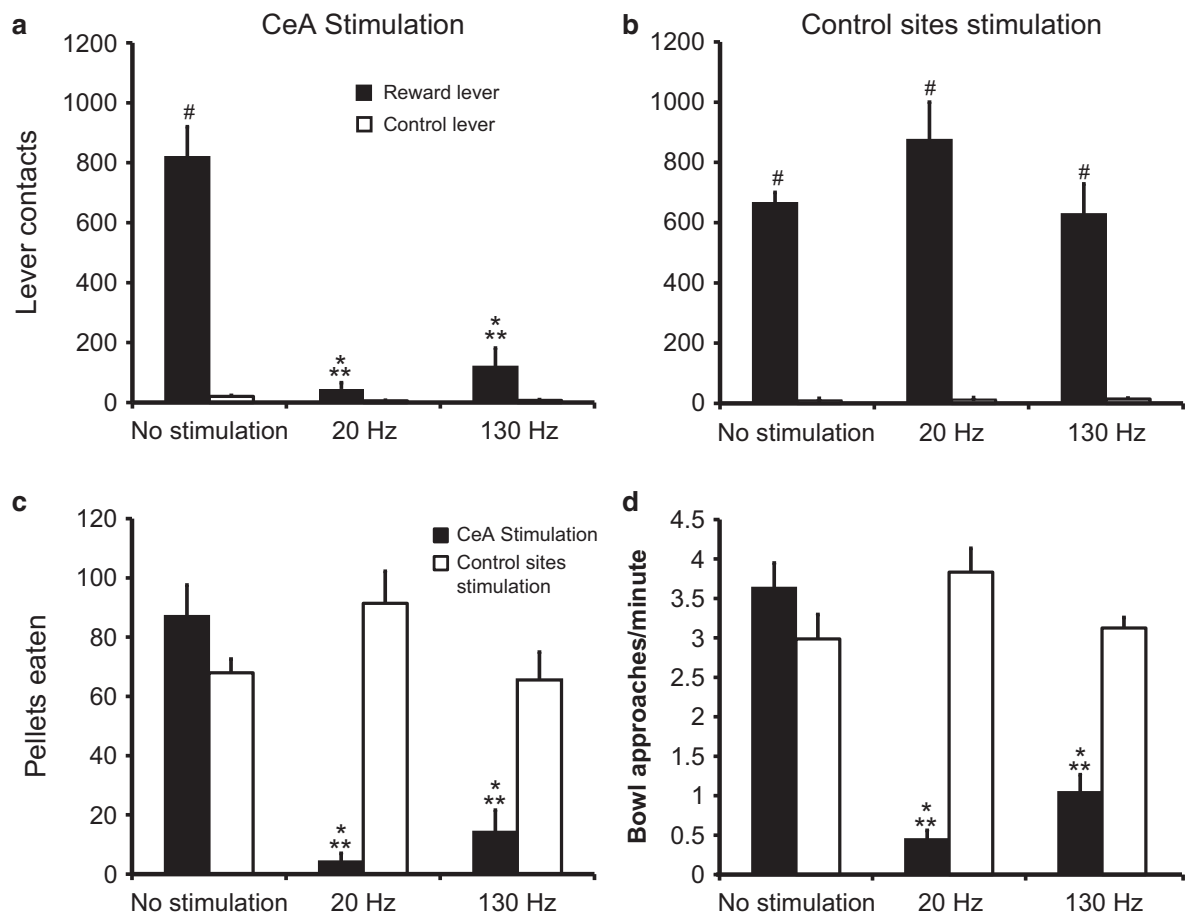

FIG. 3. DBS in the CeA decreases motivation to work for and consume sucrose pellets. (a and b) Average number of reward (black bars) and control lever (unfilled bars) contacts for no stimulation, $20 \mathrm{~Hz}$ stimulation, and $130 \mathrm{~Hz}$ stimulation. (a) Rats implanted in the CeA ( $n=7$ ). Reward lever contacts were significantly reduced during stimulation compared to no stimulation, $* P<0.001$ and compared to rats receiving stimulation in control (non-CeA) sites, $* * P<0.001$. Reward lever contacts were significantly greater than control lever contacts, ${ }^{\#} T(1)=77.000, P<0.001$ during no stimulation, but not for $20 \mathrm{~Hz}[T(1)$ $=68.000, P=0.053]$ or $130 \mathrm{~Hz}[T(1)=73.000, P=0.007]$ stimulation. (b) Rats implanted in areas outside of the CeA ( $n=4)$. There was a significant decrease in control lever contacts compared to reward lever contacts for each test condition, ${ }^{*} T(1)=78.000, P<0.001$, but no effect of stimulation. (c) Average number of sucrose pellets consumed in $30 \mathrm{~min}$ for rats implanted in the CeA (black bars) and control sites (unfilled bars). Stimulation decreased pellet consumption for rats implanted in the CeA $(* P<0.001)$ compared to no stimulation and to stimulation control sites $(* * P<0.001)$. (d) Average rate of bowl approaches. Stimulation decreased rate of bowl approaches for rats implanted in the CeA ( $P<0.001$, black bars) compared to no stimulation and to stimulation in control sites $(* * P<0.001$, unfilled bars). Error bars represent standard error.

per minute were $2.11 \pm 0.44$ (no stimulation) and $1.94 \pm 0.32$ (during stimulation) for rats implanted in control sites.

\section{Stimulation frequencies lower than $16 \mathrm{~Hz}$ had no effect}

Both 'high' frequency $(130 \mathrm{~Hz})$ and 'low' frequency $(20 \mathrm{~Hz})$ decreased operant responses and pellet consumption, that is, decreased motivational 'wanting', although we had expected $20 \mathrm{~Hz}$ to not have a 'blocking effect'. Hence, further testing was carried out in two of the CeA rats to determine if there was a threshold frequency, below which this 'blocking' effect is not observed. These two rats had previously been subjected to the same 30-min operant responding $130-\mathrm{Hz}$ and $20-\mathrm{Hz}$ tests described above. Stimulation at $16 \mathrm{~Hz}$ and $20 \mathrm{~Hz}$ decreased reward lever contacts [lever type $\mathrm{x}$ frequency: $\left.F_{5,26}=5.32 ; P=0.002\right]$ compared to no stimulation and stimulation at $2 \mathrm{~Hz}, 4 \mathrm{~Hz}$, and $10 \mathrm{~Hz}(P<0.001, \quad \mathrm{ES}=3.03$, $P<0.001, \quad \mathrm{ES}=3.27 ; \quad P=0.008, \quad \mathrm{ES}=2.95, \quad P=0.004$, $\mathrm{ES}=3.19 ; \quad P=0.001, \quad \mathrm{ES}=3.29, \quad P<0.001, \quad \mathrm{ES}=3.53$; $P=0.003, \mathrm{ES}=3.32, P=0.002, \mathrm{ES}=3.55)($ Fig. 4a). Stimulation at $16 \mathrm{~Hz}$ and $20 \mathrm{~Hz}$ reduced pellet delivery and consumption $\left[F_{5,13}=8.27 ; P=0.001,0\right.$ vs. $16 \mathrm{~Hz}: \mathrm{ES}=2.12 ; 0$ vs. $20 \mathrm{~Hz}$ : $\mathrm{ES}=2.41$ ] (Fig. 4b). Stimulation at $10 \mathrm{~Hz}$ did not differ from no stimulation $(P>0.9$, ES $<0.2)$ (Fig. 4b).

\section{CeA supports self-stimulation}

Despite the reward blocking effects of continuous stimulation, when given the opportunity, all rats would nose-poke to turn on stimulation delivered in brief $(500 \mathrm{~ms})$ bursts [reward nose-pokes vs. control nose-pokes: $\left.t_{10}=7.895, P<0.01\right]$. Self-stimulation at $130 \mathrm{~Hz}$ frequency was supported at CeA and control locations (Fig. 4c, results of self-stimulation training). Animals were then further tested at different frequencies $(20 \mathrm{~Hz}, 60 \mathrm{~Hz}$, and $130 \mathrm{~Hz})$. Twenty-Hertz frequency (Fig. 4d) supported self-stimulation, but higher frequencies generally evoked more robust responses. Stimulation at $130 \mathrm{~Hz}$ resulted in a greater number of nose-pokes compared to $60 \mathrm{~Hz}$ and $20 \mathrm{~Hz} \quad(P<0.001, \mathrm{ES}=1.03$ and $P<0.001$, $\mathrm{ES}=1.42)$. Sixty-Hertz stimulation also elicited more responses than $20 \mathrm{~Hz} \quad(P<0.001, \quad \mathrm{ES}=0.39) \quad$ (Fig. 4d) [nosepoke $\times$ frequency: $\left.F_{2,102}=19.23, P<0.001\right]$. Rats nose-poked in the rewarded hole significantly more than the unrewarded nose-poke hole $\quad(20 \mathrm{~Hz}: \quad P=<0.001, \quad \mathrm{ES}=0.15 ; \quad 60 \mathrm{~Hz}: \quad P<0.001$, $\mathrm{ES}=0.56$; and $130 \mathrm{~Hz}: P<0.001$, ES $=1.6)$ indicating that selfstimulation behavior was not a simple arousal effect.

\section{Experiment 2 (taste reactivity)}

$D B S$ attenuates hedonic value of sucrose and increase aversive reactions to taste

Taste reactivity was tested in six rats with CeA stimulation sites (Fig. 2b). Histology revealed three of the nine rats were not implanted in the CeA and were excluded from analysis. Typically, sucrose taste infusions evoked hedonic reactions. With deep brain stimulation, however, sucrose taste reactions switched to aversion $(0$ vs. $20 \mathrm{~Hz}: \quad P=0.004, \quad \mathrm{ES}=0.86 ; 0$ vs. $130 \mathrm{~Hz}: \quad P<0.001$, $\mathrm{ES}=1.30$ ) (Fig. 5a). The numbers of hedonic reactions to sucrose 
Frequency threshold

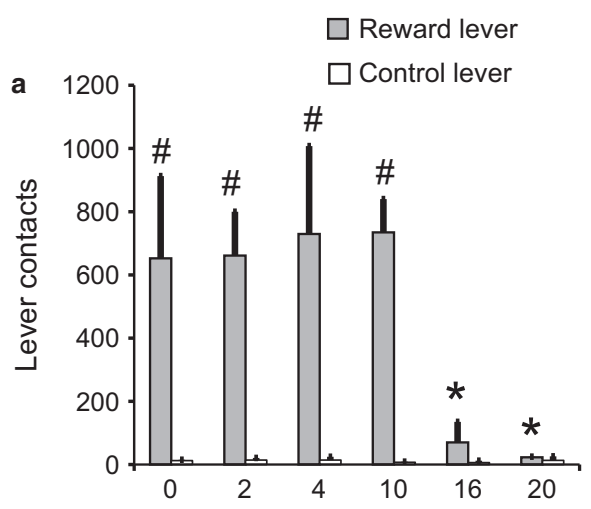

b

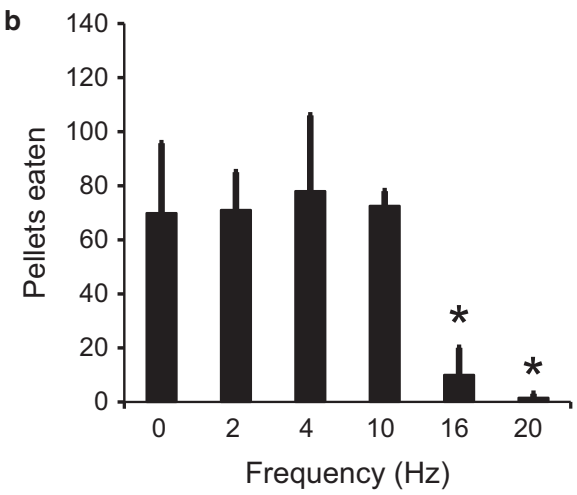

Intracranial self stimulation
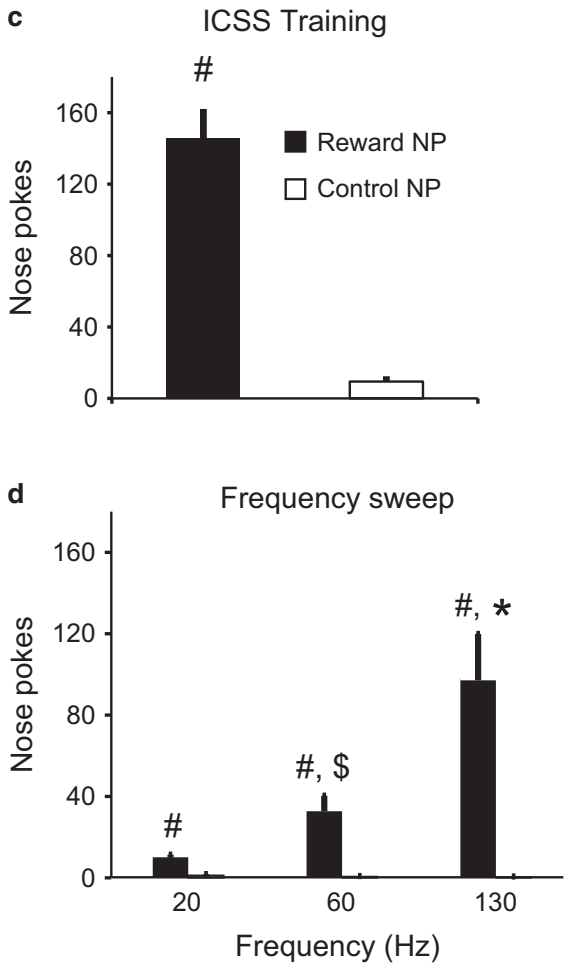

FIG. 4. (a and b) DBS at frequencies below $16 \mathrm{~Hz}$ had no effect on operant responses or pellet consumption. (a) Average number of reward (gray bars) and control lever (unfilled bars) contacts for different frequencies of stimulation and no stimulation; ${ }^{\#} P<0.001$ reward vs. control lever; ${ }^{*} P<0.01$ compared to $0 \mathrm{~Hz}, 2 \mathrm{~Hz}, 4 \mathrm{~Hz}, 10 \mathrm{~Hz} ; n=2$. (b) Average number of pellet consumption for same range of frequencies; $* P<0.01 \mathrm{compared} \mathrm{to} 0 \mathrm{~Hz}, 2 \mathrm{~Hz}, 4 \mathrm{~Hz}, 10 \mathrm{~Hz}$; $n=2$. (c) Animals implanted in the CeA and control sites all acquired self-stimulation with significant more nose-pokes into the reward nose-poke hole (black bars) compared to the control nose-poke hole (unfilled bars), ${ }^{\#} P<0.01$ at $130 \mathrm{~Hz}$ frequency, $n=11$. (d) When comparing responses at different frequencies, higher frequencies produced more robust responses $\left({ }^{*} P<0.001\right.$ for $130 \mathrm{~Hz}$ compared to $20 \mathrm{~Hz}$ and $60 \mathrm{~Hz}$; ${ }^{\$} P<0.001 \mathrm{for} 20 \mathrm{~Hz}$ vs. $\left.60 \mathrm{~Hz}, n=11\right)$; and at all 3 frequencies, animals did more nose-pokes in the reward hole (black bars) compared to the control hole $\left({ }^{\#} P<0.001\right.$, unfilled bars), NP - nose-poke. Error bars represent standard error.

decreased $(P<0.001, \mathrm{ES}=1.36$, ES $=1.78)$ while neutral reactions increased $(P<0.001, \mathrm{ES}=1.3$ for both frequencies $)$ [frequency $\times$ taste reaction $\mathrm{x}$ period: $\left.F_{4,192}=7.70, \quad P<0.001\right]$. Overall, aversive reactions outnumbered hedonic reactions $(P=0.007, \mathrm{ES}=1.15)$. Stimulation at $20 \mathrm{~Hz}$ had a similar effect with more neutral reactions compared to hedonic reactions $(P>0.001, \mathrm{ES}=2.08)$ and more aversive reactions $(P<0.001$, $\mathrm{ES}=1.80)$ (Fig. 5a). Even water, which normally produces few aversive reactions, elicited significantly more aversive reactions with $20-\mathrm{Hz}(P<0.001, \mathrm{ES}=2.92)$ and $130-\mathrm{Hz}$ stimulation $(P<0.001$, $\mathrm{ES}=1.35)$ (Fig. 5b). Not surprisingly, quinine tastes evoked even more aversive reactions than normal (Fig. 5 c) $(P<0.001, \mathrm{ES}=1.7$ for both frequencies) [frequency $\mathrm{x}$ taste reaction: $F_{4,192}=4.42$, $P=0.002$; taste reaction $\times$ period: $\left.F_{2,192}=53.60, P<0.001\right]$.

'Vacuum' reactions. During the inter-trial interval when no taste was present, rats exhibited 'vacuum' reactions, that is, facial reactions in the absence of the actual taste (Berridge \& Valenstein, 1991). These reactions in total were two or less per trial and did not differ significantly between stimulation and no stimulation (Fig. 5d) (hedonic reactions: 0 vs. $20: P=0.87, \mathrm{ES}=0.09 ; 0$ vs. 130 : $P=0.90, \quad$ ES $=0.07$; neutral reactions: 0 vs. $20: \quad P=0.48$, $\mathrm{ES}=0.22 ; 0$ vs. $130: P=0.98, \mathrm{ES}=0.006$; aversive reactions: 0 vs. 20 : $P=0.19, \mathrm{ES}=0.32$; 0 vs. $130: P=0.45$, $\mathrm{ES}=0.23$ ).

\section{Stimulation produced multiphasic firing patterns}

As a preliminary study, we studied a small number of units when possible in experiment 1 (no stimulation: 8 units, LFS: 5 units, HFS: 2 units, total 15 units) and experiment 2 (no stim: 8 units, LFS: 7 units, HFS: 4 units, total 19 units). Neurons were not silenced by stimulation which others have also shown in different brain regions (Hashimoto et al., 2003; Bar-Gad et al., 2004; Maltete et al., 2007; McCairn \& Turner, 2009; Cleary et al., 2013). Indeed, after a brief inactivation period (2-25 ms) some units actually fired faster than baseline (Fig. 6). Firing rates were assessed in the habituation period after stimulation artifacts were removed and recording time was compensated for dead time removal $(1.83 \pm 0.06 \mathrm{~ms}$ of dead time after each pulse; $n=18$ units). There was no significant rate difference during stimulation compared to the period before stimulation for either experiment $1\left[F_{2,14}=1.6, P=0.242, n=7\right.$ units] or experiment $2\left[F_{2,18}=2.627, P=0.103, n=11\right.$ units $]$. In experiment 1 , average firing rates during the first $5 \mathrm{~min}$ of habituation (before stimulation was turned on) was $5.11 \pm 1.13$ spikes $/ \mathrm{s}$ for the LFS units and dropped to $2.84 \pm 1.04$ spikes/s during the latter $5 \mathrm{~min}$ of the habituation period when stimulation was on. For the HFS units these rates were $10.38 \pm 0.38$ spikes/s and $6.27 \pm 4.86$ spikes/s respectively. In experiment 2 , comparing the mean firing rates during the first minute of the habituation period 

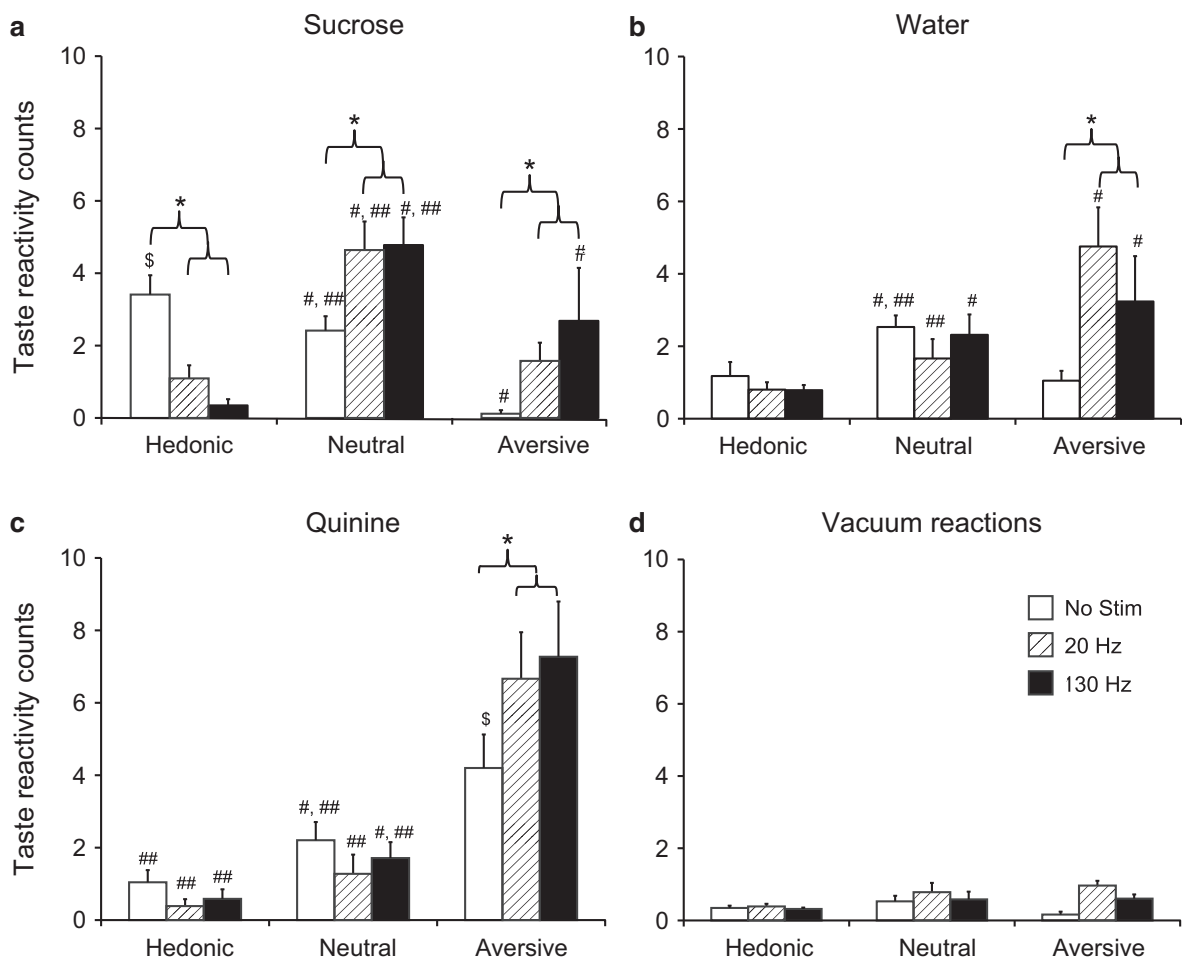

FIG. 5. DBS in the CeA decreased hedonic reactions to sucrose and also increased aversive reactions to all tastes. Plots (a-c) show the average taste reactivity counts per trial during the $10 \mathrm{~s}$ post infusion period (a) Hedonic, neutral and aversive reactions to sucrose solution for each test condition (no stimulation, 20$\mathrm{Hz}$ stimulation and $130-\mathrm{Hz}$ stimulation). Plots in (b) and (c) show hedonic, neutral and aversive reactions to water and quinine respectively. During no stimulation there were more hedonic reactions to sucrose compared to water and quinine and more aversive reactions to quinine compared to water and sucrose $\left({ }^{\$} P<0.001\right)$. For each taste: ${ }^{*} P<0.05$ compared to no stimulation within a given taste reaction, ${ }^{\#} P<0.05$ compared to hedonic reactions within a given stimulation frequency, and ${ }^{\# \#} P<0.05$ compared to aversive reactions within a given stimulation frequency. (d) Plot shows the average taste reactivity counts per trial during the $10 \mathrm{~s}$ background period between infusions (i.e. when no taste was delivered) for each test condition. These reactions were significantly fewer compared to reactions during taste $(P<0.03)$. Error bars represent standard error, $n=6$ rats.

and the second minute when stimulation was on, LFS units had $12.59 \pm 5.18$ spikes/s and $21.67 \pm 6.28$ spikes/s respectively. For HFS units, this went from $10.92 \pm 6.83$ to $7.56 \pm 2.85$ spikes/s.

Deep brain stimulation at $20 \mathrm{~Hz}$ evoked multiphasic patterns of excitations and inhibitions (Fig. 6a). Most responses began with a period of inhibition. Five units were inhibited before returning to mean pre-stimulation rates (i.e. baseline) during the period between stimulation pulses (e.g. Unit 4, Fig. 6a). One unit had a period of excitation following inhibition (Unit 2, Fig. 6a), and two units had two periods of inhibition with either an excitation (Unit 1, Fig. 6a) or a return to baseline (Unit 5, Fig. 6a) in between. Inhibition started from $\sim 2 \mathrm{~ms}$ after stimulation artifact and ranged from $2 \mathrm{~ms}$ to $22 \mathrm{~ms}$ before returning to baseline. A smaller number of units started with a period of excitation before returning to basal rates (e.g. Units 3 and 6, Fig. 6a, $n=4$ ). At $130 \mathrm{~Hz}$ DBS rates, responses were split with three units being inhibited (e.g. Unit 7, Fig. 6b) and two showing excitations followed by inhibitions (e.g. Unit 10, Fig. 6b). Inhibition was not due to stimulus artifact interference as spikes could recover between 2 and $3 \mathrm{~ms}$ after the stimulus (e.g. Units 8 and 10, Fig. 6b). There were no inherent periodic patterns evident in the control 'PSTHs' (Fig. 6 insets) indicating that the likelihood of a spike firing was independent of simply lining up spike activity at periodic intervals (Hashimoto et al., 2003).

\section{Neural-behavioral activation}

Neural units in CeA from experiment 1 were activated in association with behavioral events such as lever contact and feederclick, that is, reward delivery (Fig. 7a, first row, $n=6 / 8$ ). However, as animals rarely touched the control lever or consumed pellets with DBS on, it is challenging to assess whether neurons might still be coding behavior. Of seven units examined during stimulation, only two were, at best, weakly task responsive (Fig. 7a, second and third row).

In experiment 2, with stimulation absent, most neurons responded to sucrose (6/8) and water (5/8). A smaller number responded to quinine (3/8) (Fig. 7b, first row). Because taste reactivity testing is passive, we were able to test taste reactions while DBS was on. In contrast to high proportions of responses without stimulation, only one out of seven units responded to sucrose and quinine during $20 \mathrm{~Hz}$ stimulation and no units responded during $130 \mathrm{~Hz}$ stimulation (Fig. 7b, second and third row respectively). Deep brain stimulation at either $20 \mathrm{~Hz}$ or $130 \mathrm{~Hz}$ significantly reduced neural firing to sucrose [main effect comparing 1-s periods before and after taste onset: $\left.F_{2,37}=16.280, P<0.001\right]$. There was no effect of stimulation on neural firing to tastes of water $\left[F_{2,18}=2.698, P=0.098\right]$ or quinine $\left[F_{2,18}=0.687, P=0.518\right]$. With no stimulation, firing rates to sucrose tastes were higher than both water and quinine [main effect: $\left.F_{5,47}=5.535, P<0.001\right]$; however, with stimulation at either frequency these differences dissipated $\left[20 \mathrm{~Hz}: F_{5,41}=1.108\right.$, $\left.P=0.374 ; 130 \mathrm{~Hz}: F_{5,23}=1.503, P=0.247\right]$.

\section{Discussion}

Deep brain stimulation (DBS) of the central nucleus of the amygdala (CeA) caused animals to stop lever pressing for and 

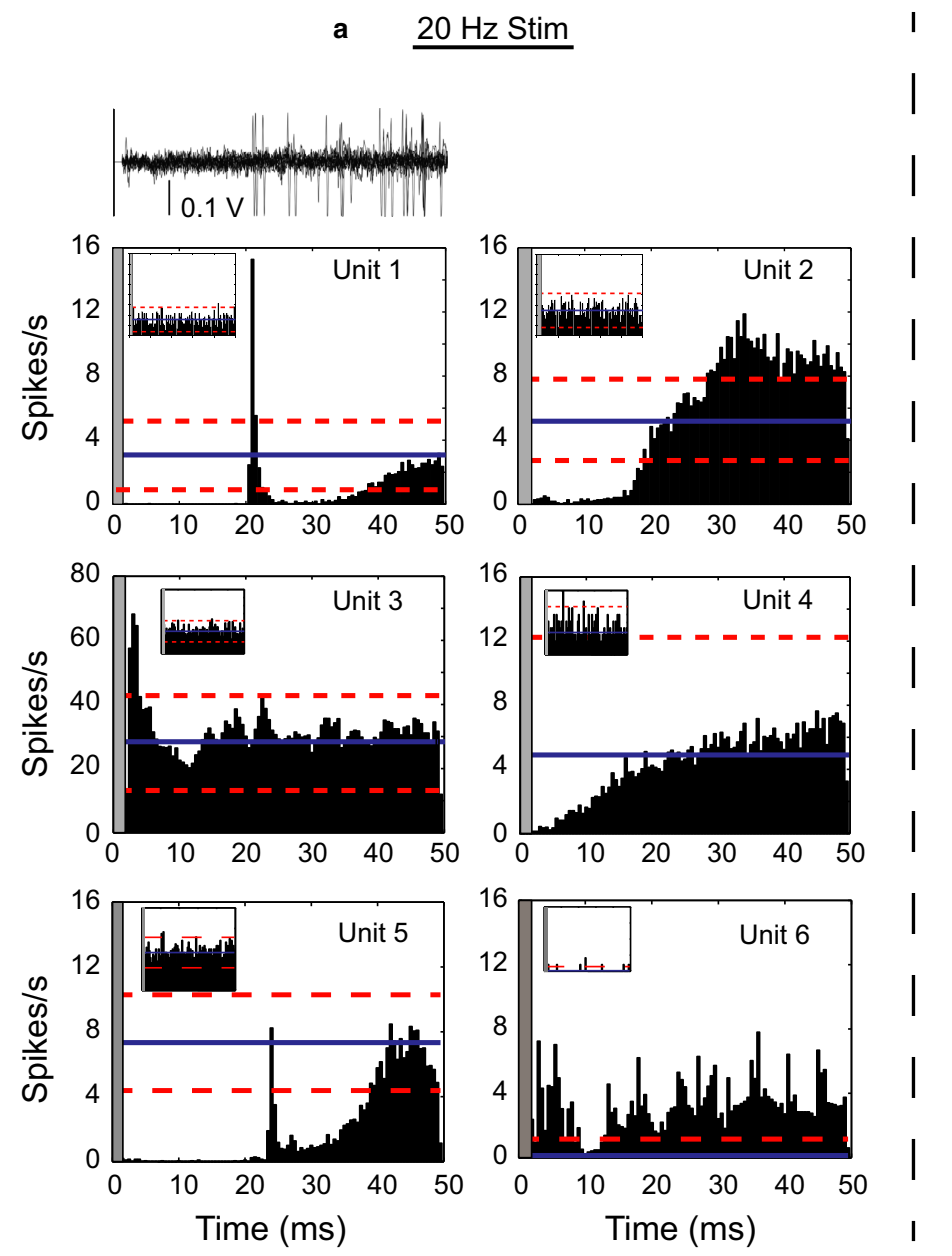

b $\quad 130 \mathrm{~Hz} \mathrm{Stim}$
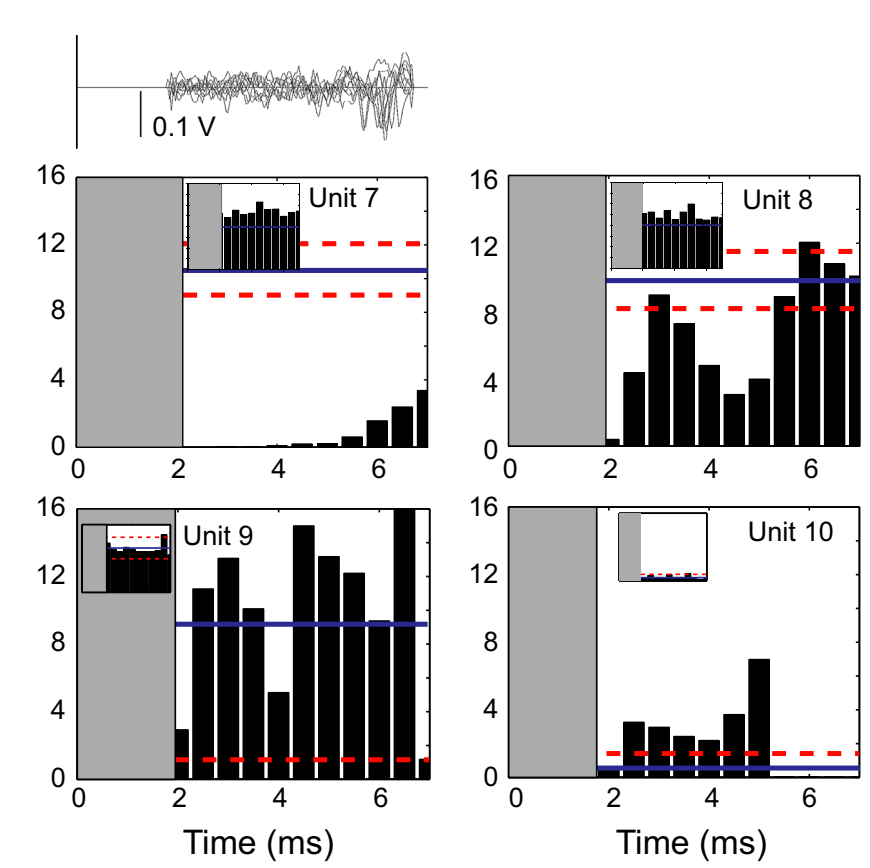

FIG. 6. Common patterns of spike activation evoked by stimulation pulses at $20 \mathrm{~Hz}$ (a) and $130 \mathrm{~Hz}$ (b) for CeA units from experiment 1 (units: $1,2,5,7$ and 8) and experiment 2 (units: 3, 4, 6, 9 and 10). Number of stimulation pulses were 42000 for units 1,2 and 5; 273000 for units 7 and $8 ; 37200$ for units 3,4 and 6; and 241800 for units 9 and 10). (a, b, Top row) 10 superimposed raw waveform sweeps from the unit in the averaged histogram shown immediately below. All histograms are aligned to the stimulus at time $=0$ for the period between stimulation pulses with 0.5 ms bins. The mean firing rate (solid line) of the unit and 2 standard deviations from the mean (dashed lines) are indicated on the histogram plots. The gray areas beginning on the left of each histogram mark the period of stimulus artifact dead time during which no spikes could be detected. The inset histograms computed by alignment to 'sham' stimulation pulses in the period before stimulation (in the same units) show no inherent background spike activity pattern (for experiment 1: 6000 stimulation pulses for $20 \mathrm{~Hz}$ and 39000 for $130 \mathrm{~Hz}$; experiment 2: 1200 for $20 \mathrm{~Hz}$ and 7000 for $130 \mathrm{~Hz}$ ). At $130 \mathrm{~Hz}$, the inter-stimulus interval is $7.6 \mathrm{~ms}$; hence, by necessity $x$ axes for the right graphs are shorter. [Color version of figure available online].

consuming sucrose pellet rewards. Taste reactivity tests revealed increased 'disliking' reactions to palatable, neutral, and aversive tastes. Surprisingly, DBS presented in brief pulses in the same location under the animals' control resulted in vigorous self-stimulation. Overall, neural recordings during stimulation showed smaller proportions of responsive neurons to reward-related events.

\section{LFS at $20 \mathrm{~Hz}$ as effective as HFS at $130 \mathrm{~Hz}$}

Therapeutic effects of DBS for Parkinson's disease, essential tremor and dyskinesia typically occur at frequencies greater than $90 \mathrm{~Hz}$. Conversely, lower frequencies $(<50 \mathrm{~Hz})$ have been reported to have no therapeutic benefit or even worsen symptoms (Rizzone et al., 2001; Moro et al., 2002; Kuncel et al., 2007). Thus, we anticipated that stimulation at $130 \mathrm{~Hz}$ would 'block' while $20 \mathrm{~Hz}$ stimulation might 'activate' the circuit and behavior. Surprisingly though, both frequencies decreased pellet delivery and consumption and also increased aversive reactions to tastes.
CeA units have very low firing rates, typically less than $5 \mathrm{~Hz}$ (Collins \& Paré, 1999; Rosenkranz \& Grace, 1999). So, for CeA, $20 \mathrm{~Hz}$ might effectively be a 'high' frequency that overwhelms the very low intrinsic frequency of $\mathrm{CeA}$ neurons. Frequency effects depend on the type of structure (Dostrovsky \& Lozano, 2002) and the intrinsic frequency of neurons being stimulated (Grill et al., 2004). Our frequency sweep study did show that there was no effect of DBS at frequencies $10 \mathrm{~Hz}$ and lower (Fig. 4a and b) with a surprising drop off between 10 and $16 \mathrm{~Hz}$. We did not test frequencies between $10 \mathrm{~Hz}$ and $16 \mathrm{~Hz}$ so it is possible that between those values there are frequencies that might produce an intermediate effect, although others have reported abrupt transitions with DBS (Wu et al., 2001). This threshold effect we observed could be due to an intrinsic oscillation frequency between $10 \mathrm{~Hz}$ and $16 \mathrm{~Hz}$ above which the stimulation frequency is 'fast' enough to overwhelm the circuit and below which normal neural responses to reward events can pass through. Most neurons in the medial nucleus of the CeA are referred to as 'late firing', that is, in response to current injection the membrane potential depolarizes slowly over hundreds of 
No Stim

a Experiment 1

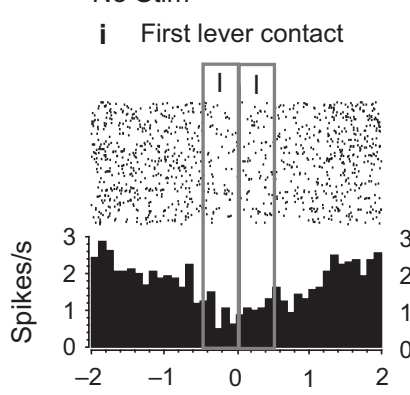

ii Feederclick

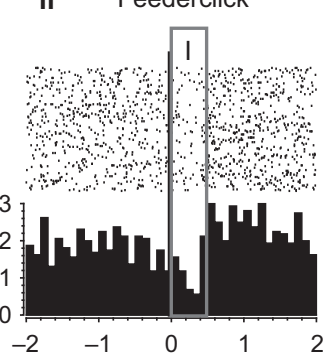

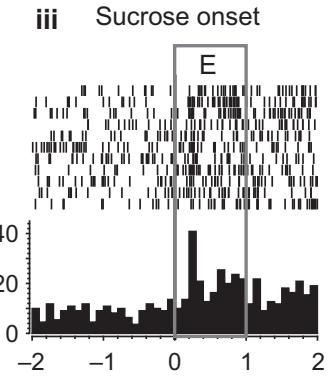

b Experiment 2
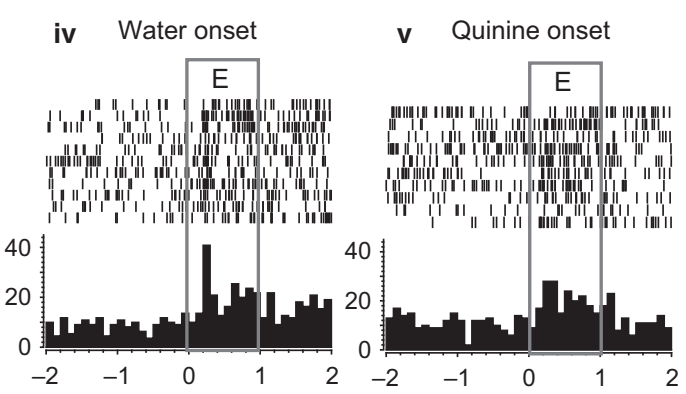

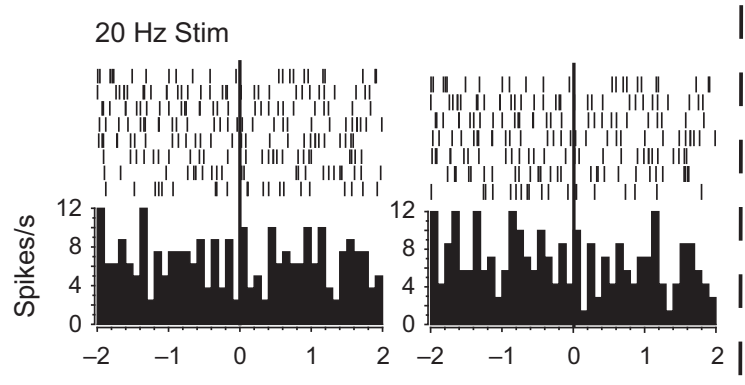

I
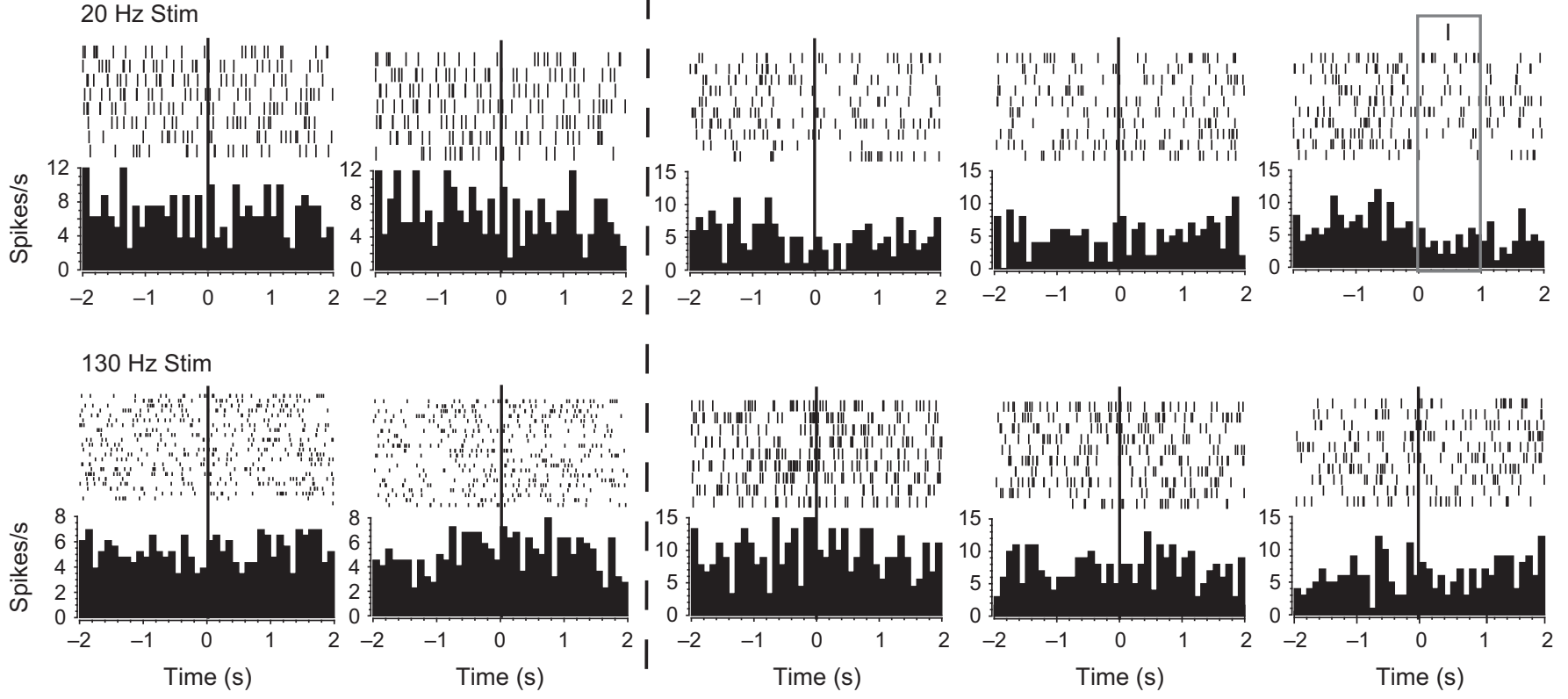

FIG. 7. Fewer CeA unit responses during stimulation to task events. Representative PETHs and raster plots for (a) experiment 1 and (b) experiment 2 during no stimulation (top row), $20 \mathrm{~Hz}$ stimulation (middle row), and $130 \mathrm{~Hz}$ stimulation (bottom row) for different events (columns i-v). Plots show unit activity up to $2 \mathrm{~s}$ before and after the event of interest which is centered at 0 . Each mark in a raster indicates a spike and each horizontal line is a trial, with consecutive trials going from top to bottom. Histograms (bin width $=100 \mathrm{~ms}$ ) show the average firing rate across all trials. Inhibition (I) and excitation (E) responses to particular events are highlighted by gray rectangles. Responses are with respect to the 5-s period just before the lever extended for experiment 1 (a) and the 1 -s period before the taste onset for experiment 2 (b).

milliseconds (Martina et al., 1999). These membrane dynamics could contribute to the frequency dependence and threshold effects for DBS-like stimulation.

\section{Is this a decrease in 'wanting' only?}

Our results showed that DBS decreased food consumption and the number of operant responses on the reward lever. But is this decrease in pellet delivery and consumption only due to a decrease in motivation or also due to a change in palatability of the food? During DBS some rats would put the pellets in their mouths and then spit them out, suggesting they still 'wanted' the pellets but no longer found them palatable. Other rats remained by the food bowl or the lever and even occasionally delivered more pellets, while some completely ignored the lever and food bowl. Histology did not reveal a difference in electrode locations that could account for these individual differences; but, the $\mathrm{CeA}$ is a heterogeneous nucleus (Swanson \& Petrovich, 1998) and it is possible that differences in the underlying tissue and current spread could result in different neuronal components being activated. Furthermore, stimulation could be impacting both upstream and downstream structures by activating afferent and efferent axons as well as fibers of passage (Okun, 2012). Hence, all of these factors could account for these slight differences in behavior.

CeA stimulation not only decreased 'wanting' of food rewards, but also affected 'liking' responses. In our taste reactivity study, stimulation resulted in decreased 'liking' and increased 'disliking' reactions to tastes. Riley \& King (2013) also reported increases in aversive reactions to tastes with $\mathrm{CeA}$ stimulation. In addition, we observed slightly more neutral and aversive reactions in the absence of tastes (although not significant). Even in our operant response experiment, rats would sometimes produce gaping reactions. Hence, DBS in the CeA could be creating a general aversive state. Studies have reported increases in aversive reactions to quinine (Touzani et al., 1997) and reduction in quinine consumption (Kemble et al., 1979) with CeA lesions and it has been proposed that CeA may play a role in modulating food intake based on taste palatability (Kemble et al., 1979; Touzani et al., 1997; Li et al., 2012). However, others have reported no change in aversive reactions to disliked tastes with CeA manipulations (Galaverna et al., 1993; Seeley 
et al., 1993; Mahler \& Berridge, 2011; Robinson et al., 2014). These differences could be due to different types of manipulations used and the heterogeneity of the structure itself. A recent study by Cai et al. (2014) has identified populations of neurons in the lateral subdivision of the CeA that play important, but opposite roles in influencing feeding.

The CeA is a site that supports self-stimulation (Wurtz \& Olds, 1963). We also found that animals would self-stimulate if the same stimulation parameters were used in short bursts $(0.5 \mathrm{~s})$ of stimulation. Both 20 and $130 \mathrm{~Hz}$ stimulation were effective although the higher frequency was more robust as other studies have shown (Arvanitogiannis \& Shizgal, 2008). Interestingly, although animals would vigorously engage in CeA self-stimulation they would sometimes produce 'aversive' gapes similar to that was observed in the operant responding and taste reactivity experiments, which may again suggest stimulation is also triggering an aversive response. It is also possible that this short burst of stimulation could be triggering motivational circuits and hence be 'wanted' although not necessarily 'liked'. It should be noted that Robinson et al. (2014) showed that animals would not self-stimulate with optogenetic activation of CeA. These differences could be due in part to the duration of the stimulation train used ( $8 \mathrm{~s}$ vs. $0.5 \mathrm{~s}$ ) and the type of stimulation (optogenetic vs. electrical) with electrical stimulation likely activating axons and cells in more widespread regions than optogenetic stimulation. Finally, the intrinsic membrane properties of $\mathrm{CeA}$ neurons mentioned above (Martina et al., 1999) with regard to thresholds may also confer different consequences for short and long bursts of stimulation.

Perhaps it is not a change in palatability in the food per se that is impacting food consumption. Feeding behavior and motivational mechanisms behind feeding can be modulated by many signals (Saper et al., 2002; Sternson, 2013). Need-based states and neurons and/or chemicals that signal these states can impact motivation to seek out and consume food. This motivation could stem from the desire to reduce the negative valence due to hunger (Sternson, 2013). Agouti related protein (AGRP)-expressing neurons in the hypothalamus are activated by energy deficit resulting in increased feeding behavior. However, continuous photo-stimulation of these neurons decreased instrumental responding for food over the course of days (Betley et al., 2015). Betley et al. hypothesized that as these neurons kept signaling a negative state, the animals stopped responding as their actions failed to reduce this state. We also saw decreased instrumental responding with DBS, so it is possible that something similar could be occurring here, although we saw decreased responding within seconds and minutes, compared to over a day in their study. Testing could be done to determine if these neurons are being activated with the type of stimulation used in our study. Again, given the differences in optogenetic vs. electrical stimulation, DBS may be decreasing consumption by different mechanisms. Perhaps DBS is inhibiting motivational or hunger circuits. Further discussion on this possible inhibitory mechanism of DBS is discussed below.

More directly, within the $\mathrm{CeA}$ itself, in the lateral subdivision, there is a subpopulation of neurons $\left(\mathrm{PKC}-\delta^{+}\right)$that are activated by multiple anorexigenic signals. Unpalatable food, but also other anorexigenic signals that trigger satiation or visceral malaise, can lead to decrease in food consumption. Optogenetic activation of these neurons resulted in decreased food consumption in both fooddeprived and fed mice (Cai et al., 2014). Hence, we could be also be activating those neurons resulting in decreased motivation for food rewards and increased aversive responses. (Note, this does not rule out a decrease in perceived palatability as these neurons respond to multiple signals including bitter tastants).
Inactivation of the $\mathrm{CeA}$ has also been shown to decrease dopamine baseline levels and dopamine efflux in the nucleus accumbens (Ahn \& Phillips, 2002). DBS could be inactivating the CeA, resulting in a similar mechanism. Future studies could look at the effects of DBS in the CeA with 6-OHDA lesions to tease out the role of dopamine in this (something similar could be done for opioid antagonists). Howland and colleagues (2002) did observe no significant change in dopamine levels during a short burst of stimulation delivered to the $\mathrm{CeA}(10 \mathrm{~s}, 300 \mu \mathrm{A})$. However, they did indicate that their microdiaylsis technique may be too slow to record faster changes in dopamine. Interestingly, at higher amplitudes (up to $800 \mu \mathrm{A}$ ), they reported chewing behavior which could be similar to the increased mouth movements we observed with stimulation (Howland et al., 2002).

Our data show that both 'wanting' and 'liking' of food rewards are affected with stimulation, although more research is needed to determine if both systems are being activated or if one is directly impacting the other (i.e. are animals not eating because the food is no longer palatable or does the animal neither 'like' nor 'want' the food). Further testing is needed to determine whether it is a change in palatability or a more physiological reason for this decrease in 'wanting'. As part of that, it also should be determined if DBS in the $\mathrm{CeA}$ is creating a general aversive state, by exploring DBS's effects on other rewards such as sex and drugs.

\section{Is this decreased feeding due to fear and anxiety?}

Our results could possibly be due to an increase in fear and anxiety. The CeA does play a role in fear and anxiety (Maren \& Fanselow, 1996; Pitkanen et al., 1997; Tye et al., 2011). Although we cannot completely rule this out, there is some evidence that suggests this is not the case. In experiment 1 , rats receiving stimulation in the CeA would still cross the center of the chamber, rear, groom, and interact with the food bowl and levers (even sniffing and occasionally nibbling at the bowl). We did not see any significant differences in chamber crossing and rearing between rats being stimulated in the $\mathrm{CeA}$ vs. in our control sites. Some of the animals receiving stimulation in the $\mathrm{CeA}$ did deliver and eat pellets throughout the session (although significantly less than when stimulation was off). In both experiments, we observed gapes and forelimb flails and occasional defensive treading during stimulation, but we did not see any obvious signs of freezing. We also did not observe any obvious changes in autonomic systems such as changes in respiration rate (though this was not directly measured). Optogenetic excitation of the CeA has also been reported to have anxiolytic effects (Tye et al., 2011; Cai et al., 2014). However, more tests should be carried out before we can rule this out completely.

\section{Mechanism of DBS?}

We found that DBS in the CeA produced similar behavioral effects (decrease in food and liquid consumption and increased aversive reactions) as seen with lesioning or inactivation of the CeA. The various manipulations included the use of fiber-sparing lesioning agents such as kainic acid (Hajnal et al., 1992) and ibotenic lesions (Touzani et al., 1997); as well as, lidocaine (Ahn \& Phillips, 2002), muscimol (Will et al., 2009) and cauterization of tissue (Kemble et al., 1979; Galaverna et al., 1993). Ahn \& Phillips (2002) also noted that their CeA-lesioned rats would put food in their mouths but then discard the partially eaten food, which was a behavior we observed in some of our rats during stimulation. However, our findings with neural recordings indicate that DBS did not just silence 
neurons. We found that stimulation in the $\mathrm{CeA}$ induced multiphasic patterns of firing that could represent loose entrainment of unit activity to stimulation. Units were more likely to fire at specific latencies from stimulation pulse onset (Fig. 6), suggesting a form of entrainment. These responses have been seen in units recorded near the site of stimulation (Bar-Gad et al., 2004; Carlson et al., 2010; Cleary et al., 2013) and at distant targets (Hashimoto et al., 2003; Maltete et al., 2007; McCairn \& Turner, 2009). Stimulation also seems to be disrupting CeA encoding of reward. Even after accounting for artifact dead time, we saw fewer and weaker responses to reward-related events in both studies.

It is argued that DBS could be imposing its own pattern that is no longer meaningful to the underlying circuit (McIntyre et al., 2004; Garcia et al., 2005a,b; Johnson \& McIntyre, 2008). In a sense, creating an 'information lesion' (Grill et al., 2004) and disrupting information flow (whether normal or pathological) in the circuit. Therefore, stimulation could be inducing its own pattern of firing in the $\mathrm{CeA}$ and disrupting neural coding for food rewards and taste palatability, making things more aversive.

Histological verification from our study (Fig. 2) showed that the majority of electrodes were in the medial division of the CeA (CeM). Some of the primary connections to the CeM are from the lateral subdivision of the CeA (CeL) which is GABAergic. The output of the CeM is also primarily GABAergic (Swanson \& Petrovich, 1998). Given some of the similar responses between lesion and activating studies and our results and the likelihood of DBS activating axons, it is possible that the stimulation could be activating axons from the CeL releasing GABA into the CeM and thus inhibiting it. This, in turn could lead to disinhibition of downstream structures ultimately decreasing food consumption and motivation. For example, axons from the $\mathrm{CeA}$ have been proposed to synapse onto a local GABAergic interneuron network in the ventral tegmental area (VTA). Disinhibition of these interneurons could result in increased inhibition of the VTA and thus inhibition of dopaminergic neurons projecting to the nucleus accumbens (Howland et al., 2002). Taste pathways entering into the $\mathrm{CeA}$ could also be activated ultimately resulting in increased aversion possibly although opioid activation (Ricardo \& Koh, 1978; Mahler \& Berridge, 2011). More targeted stimulation such as optogenetic stimulation (Gradinaru et al., 2009) could be used to shed more light on these different unit responses.

\section{Conclusion}

Our results demonstrate that the $\mathrm{CeA}$ is a very effective target for blocking 'wanting' and 'liking' of food rewards. Both $20 \mathrm{~Hz}$ and $130 \mathrm{~Hz}$ stimulation resulted in significant decreases in working for the delivery of a food reward, consumption of that reward but also increased aversive responses to sweet, neutral, and bitter tastes. Stimulation produced multiphasic patterns in CeA units and resulted in fewer responsive units to reward-related events. Questions remain whether stimulation is independently affecting both 'wanting' and 'liking' circuits, or only directly modulating 'liking' circuits which in turn is affecting 'wanting'. Furthermore, if stimulation is creating a general aversive state one wonders if this extends to other rewards and it presents obstacles to the potential clinical utility of DBS for blocking reward dysfunction. Although DBS in the CeA was very effective at decreasing motivation for food rewards and thus food consumption, there were accompanying aversive reactions. Fewer aversive reactions were seen with $20-\mathrm{Hz}$ stimulation, so it is possible that stimulation parameters could be optimized. Further testing is needed to assess possible side effects as well as long term effects of DBS in the CeA.

\section{Conflict of interests}

The authors declare no competing financial interests.

\section{Supporting Information}

Additional supporting information can be found in the online version of this article:

Data S1. Current density calculations and volume of tissue activated due to stimulation.

Fig. S1. Mapping study for each rat showing the actual electrode paths (verified with histology) and the number of sucrose pellets consumed in $10 \mathrm{~min}$ tests at different depths.

Movie S1. Clip of a rat (video-recorded from below) implanted in the CeA performing the operant responding task from experiment 1 , first in the absence of stimulation and then during $20-\mathrm{Hz}$ stimulation.

\section{Acknowledgements}

This study was supported by NIDA DA017752 (JWA), NIH T32 NS02222-28 training grant, and University of Michigan internal funding. The authors thank Dr. Paul Meyer for his helpful discussion and experimental design. We thank Andrew Klein, Steve Zekany and Nathan Patel for their technical help and data analysis and Marc Bradshaw for his technical assistance. We also thank Dr. Brady West and the Center for Statistical Consulting and Research, University of Michigan, Ann Arbor, MI 48109 for their help with statistical analysis.

\section{Abbreviations}

AA, anterior amygdaloid area; ASt, amygdalostriatal transition area; BLA basolateral amygdaloid nucleus; BMA, basomedial amygdaloid nucleus; $\mathrm{CeA}$, central nucleus of the amygdala; $\mathrm{CeC}$, central nucleus of the amygdala, capsular part; CeL, central nucleus of the amygdala, lateral division; CeM, central nucleus of the amygdala, medial division; $\mathrm{CPu}$, caudate putamen; DBS, deep brain stimulation; DEn, dorsal endopiriform nucleus; EA, extended amygdala; FR, fixed ratio; GP, globus pallidus; HFS, high-frequency stimulation; I, intercalated nuclei of the amygdala; IM, intercalated amygdaloid nucleus main part; LFS, low frequency stimulation; MCPO, magnocellular preoptic nucleus; MeAD, medial amygdaloid nucleus, anterior dorsal; MePD, medial amygdaloid nucleus posterodorsal part; VEn, ventral endopiriform nucleus; VP, ventral pallidum.

\section{References}

Ahn, S. \& Phillips, A.G. (2002) Modulation by central and basolateral amygdalar nuclei of dopaminergic correlates of feeding to satiety in the rat nucleus accumbens and medial prefrontal cortex. J. Neurosci., 22, 1095810965.

Arvanitogiannis, A. \& Shizgal, P. (2008) The reinforcement mountain: allocation of behavior as a function of the rate and intensity of rewarding brain stimulation. Behav. Neurosci., 122, 1126-1138.

Bar-Gad, I., Elias, S., Vaadia, E. \& Bergman, H. (2004) Complex locking rather than complete cessation of neuronal activity in the globus pallidus of a 1-methyl-4-phenyl-1,2,3,6-tetrahydropyridine-treated primate in response to pallidal microstimulation. J. Neurosci., 24, 7410-7419.

Baxter, M.G. \& Murray, E.A. (2002) The amygdala and reward. Nat. Rev. Neurosci., 3, 563-573.

Berridge, K.C. (2000) Measuring hedonic impact in animals and infants: microstructure of affective taste reactivity patterns. Neurosci. Biobehav. R., 24, 173-198.

Berridge, K.C. \& Valenstein, E.S. (1991) What psychological process mediates feeding evoked by electrical stimulation of the lateral hypothalamus? Behav. Neurosci., 105, 3-14.

Betley, J.N., Xu, S., Cao, Z.F., Gong, R., Magnus, C.J., Yu, Y. \& Sternson, S.M. (2015) Neurons for hunger and thirst transmit a negative-valence teaching signal. Nature, 521, 180-185.

Cai, H., Haubensak, W., Anthony, T.E. \& Anderson, D.J. (2014) Central amygdala PKC-delta(+) neurons mediate the influence of multiple anorexigenic signals. Nat. Neurosci., 17, 1240-1248. 
Carlson, J.D., Cleary, D.R., Cetas, J.S., Heinricher, M.M. \& Burchiel, K.J. (2010) Deep brain stimulation does not silence neurons in subthalamic nucleus in Parkinson's patients. J. Neurophysiol., 103, 962-967.

Chang, J.Y. (2004) Brain stimulation for neurological and psychiatric disorders, current status and future direction. J. Pharmacol. Exp. Ther., 309, 17.

Cleary, D.R., Raslan, A.M., Rubin, J.E., Bahgat, D., Viswanathan, A., Heinricher, M.M. \& Burchiel, K.J. (2013) Deep brain stimulation entrains local neuronal firing in human globus pallidus internus. J. Neurophysiol., 109, 978-987.

Collins, D.R. \& Paré, D. (1999) Reciprocal changes in the firing probability of lateral and central medial amygdala neurons. J. Neurosci., 19, 836-844.

DiFeliceantonio, A.G. \& Berridge, K.C. (2012) Which cue to 'want'? opioid stimulation of central amygdala makes goal-trackers show stronger goaltracking, just as sign-trackers show stronger sign-tracking. Behav. Brain Res., 230, 399-408.

Doshi, P.K. (2011) Long-term surgical and hardware-related complications of deep brain stimulation. Stereot. Funct. Neuros., 89, 89-95.

Dostrovsky, J.O. \& Lozano, A.M. (2002) Mechanisms of deep brain stimulation. Movement Disord., 17, S63-S68.

Galaverna, O.G., Seeley, R.J., Berridge, K.C., Grill, H.J., Epstein, A.N. \& Schulkin, J. (1993) Lesions of the central nucleus of the amygdala. I: effects on taste reactivity, taste aversion learning and sodium appetite. Behav. Brain Res., 59, 11-17.

Garcia, L., D'Alessandro, G., Bioulac, B. \& Hammond, C. (2005a) High-frequency stimulation in Parkinson's disease: more or less? Trends Neurosci. 28, 209-216.

Garcia, L., D’Alessandro, G., Fernagut, P.O., Bioulac, B. \& Hammond, C. (2005b) Impact of high-frequency stimulation parameters on the pattern of discharge of subthalamic neurons. J. Neurophysiol., 94, 3662-3669.

Gradinaru, V., Mogri, M., Thompson, K.R., Henderson, J.M. \& Deisseroth, K. (2009) Optical deconstruction of parkinsonian neural circuitry. Science, 324, 354-359.

Greenberg, B.D., Gabriels, L.A., Malone, D.A., Rezai, A.R., Friehs, G.M., Okun, M.S., Shapira, N.A., Foote, K.D. et al. (2010) Deep brain stimulation of the ventral internal capsule/ventral striatum for obsessive-compulsive disorder: worldwide experience. Mol. Psychiatr., 15, 64-79.

Grill, H.J. \& Norgren, R. (1978) Taste reactivity test.1. Mimetic responses to gustatory stimuli in neurologically normal rats. Brain Res., 143, 263-279.

Grill, W.M., Snyder, A.N. \& Miocinovic, S. (2004) Deep brain stimulation creates an informational lesion of the stimulated nucleus. NeuroReport, $\mathbf{1 5}$ 1137-1140.

Gubellini, P., Salin, P., Kerkerian-Le Goff, L. \& Baunez, C. (2009) Deep brain stimulation in neurological diseases and experimental models: from molecule to complex behavior. Prog. Neurobiol., 89, 79-123.

Haber, S.N. \& Brucker, J.L. (2009) Cognitive and limbic circuits that are affected by deep brain stimulation. Front Biosci., 14, 1823-1834.

Hajnal, A., Sandor, P., Jando, G., Vida, I., Czurko, A., Karadi, Z. \& Lenard, L. (1992) Feeding disturbances and EEG activity changes after amygdaloid kainate lesions in the rat. Brain Res. Bull., 29, 909-916.

Halpern, C.H., Samadani, U., Litt, B., Jaggi, J.L. \& Baltuch, G.H. (2008) Deep brain stimulation for epilepsy. Neurotherapeutics, 5, 59-67.

Hashimoto, T., Elder, C.M., Okun, M.S., Patrick, S.K. \& Vitek, J.L. (2003) Stimulation of the subthalamic nucleus changes the firing pattern of pallidal neurons. J. Neurosci., 23, 1916-1923.

Heldmann, M., Berding, G., Voges, J., Bogerts, B., Galazky, I., Muller, U., Baillot, G., Heinze, H.J. et al. (2012) Deep brain stimulation of nucleus accumbens region in alcoholism affects reward processing. PLoS One, 7, 1-7.

Howland, J.G., Taepavarapruk, P. \& Phillips, A.G. (2002) Glutamate receptor-dependent modulation of dopamine efflux in the nucleus accumbens by basolateral, but not central, nucleus of the amygdala in rats. J. Neurosci., 22, 1137-1145

Johnson, M.D. \& McIntyre, C.C. (2008) Quantifying the neural elements activated and inhibited by globus pallidus deep brain stimulation. J. Neurophysiol., 100, 2549-2563.

Kemble, E.D., Studelska, D.R. \& Schmidt, M.K. (1979) Effects of central amygdaloid nucleus lesions on ingestion, taste reactivity, exploration and taste aversion. Physiol. Behav., 22, 789-793.

Kringelbach, M.L., Jenkinson, N., Owen, S.L. \& Aziz, T.Z. (2007) Translational principles of deep brain stimulation. Nat. Rev. Neurosci., 8, 623635.

Kuncel, A.M., Cooper, S.E., Wolgamuth, B.R. \& Grill, W.M. (2007) Amplitude- and frequency-dependent changes in neuronal regularity paralle changes in tremor with thalamic deep brain stimulation. IEEE T Neur. Sys. Reh., 15, 190-197.
Li, J., Yan, J., Chen, K., Lu, B., Wang, Q., Yan, W. \& Zhao, X. (2012) Lesions of the central nucleus of the amygdala decrease taste threshold for sodium chloride in rats. Brain Res. Bull., 89, 8-15.

Liu, L.D., Prescott, I.A., Dostrovsky, J.O., Hodaie, M., Lozano, A.M. \& Hutchison, W.D. (2012) Frequency-dependent effects of electrical stimulation in the globus pallidus of dystonia patients. J. Neurophysiol., 108, 517

Lozano, A.M., Mayberg, H.S., Giacobbe, P., Hamani, C., Craddock, R.C. \& Kennedy, S.H. (2008) Subcallosal cingulate gyrus deep brain stimulation for treatment-resistant depression. Biol. Psychiat., 64, 461-467.

Mahler, S.V. \& Berridge, K.C. (2009) Which cue to "want?" central amygdala opioid activation enhances and focuses incentive salience on a prepotent reward cue. J. Neurosci., 29, 6500-6513.

Mahler, S.V. \& Berridge, K.C. (2011) What and when to "want"? Amygdala-based focusing of incentive salience upon sugar and sex. Psychopharmacology, 221, 407-426.

Maltete, D., Jodoin, N., Karachi, C., Houeto, J.L., Navarro, S., Cornu, P., Agid, Y. \& Welter, M.L. (2007) Subthalamic stimulation and neuronal activity in the substantia nigra in Parkinson's disease. J. Neurophysiol., 97, 4017-4022.

Maren, S. \& Fanselow, M.S. (1996) The amygdala and fear conditioning: has the nut been cracked? Neuron, 16, 237-240.

Martina, M., Royer, S. \& Pare, D. (1999) Physiological properties of central medial and central lateral amygdala neurons. J. Neurophysiol., 82, $1843-$ 1854.

Mayberg, H., Lozano, A., Voon, V., McNeely, H., Seminowicz, D., Hamani, C., Schwalb, J. \& Kennedy, S. (2005) Deep brain stimulation for treatment-resistant depression. Neuron, 45, 651-660.

McCairn, K.W. \& Turner, R.S. (2009) Deep brain stimulation of the globus pallidus internus in the parkinsonian primate: local entrainment and suppression of low-frequency oscillations. J. Neurophysiol., 101, 19411960.

McDonald, A.J. \& Augustine, J.R. (1993) Localization of GABA-like immunoreactivity in the monkey amygdala. Neuroscience, 52, 281-294.

McIntyre, C.C., Grill, W.M., Sherman, D.L. \& Thakor, N.V. (2004) Cellular effects of deep brain stimulation: model-based analysis of activation and inhibition. J. Neurophysiol., 91, 1457-1469.

Melega, W.P., Lacan, G., Gorgulho, A.A., Behnke, E.J. \& De Salles, A.A. (2012) Hypothalamic deep brain stimulation reduces weight gain in an obesity-animal model. PLoS One, 7, 1-9.

Moro, E., Esselink, R., Xie, J., Hommel, M., Benabid, A. \& Pollak, P. (2002) The impact on Parkinson's disease of electrical parameter settings in STN stimulation. Neurology, 59, 706-713.

Nowak, L.G. \& Bullier, J. (1998) Axons, but not cell bodies, are activated by electrical stimulation in cortical gray matter. I. Evidence from chronaxie measurements. Exp. Brain Res., 118, 477-488.

Okun, M.S. (2012) Deep-brain stimulation for Parkinson's disease. New Engl. J. Med., 367, 1529-1538.

Paxinos, G. \& Watson, C. (2007) The Rat Brain in Stereotaxic Coordinates. Elsevier Inc., Amsterdam, the Netherlands.

Pitkanen, A., Savander, V. \& LeDoux, J. (1997) Organization of intra-amygdaloid circuitries in the rat: an emerging framework for understanding functions of the amygdala. Trends Neurosci., 20, 517-523.

Plonsey, R. \& Barr, R. C. (2007) Bioelectricity A Quantitative Approach. Springer Science+Business Media, LLC, New York, NY, pp. 28-29.

Price, J.L. \& Amaral, D.G. (1981) An autoradiographic study of the projections of the central nucleus of the monkey amygdala. J. Neurosci., 1, $1242-1259$.

Ranck, J.B. Jr (1975) Which elements are excited in electrical stimulation of mammalian central nervous system: a review. Brain Res., 98, $417-$ 440.

Ricardo, J.A. \& Koh, E.T. (1978) Anatomical evidence of direct projections from the nucleus of the solitary tract to the hypothalamus, amygdala, and other forebrain structures in the rat. Brain Res., 153, 1-26.

Riley, C.A. \& King, M.S. (2013) Differential effects of electrical stimulation of the central amygdala and lateral hypothalamus on fos-immunoreactive neurons in the gustatory brainstem and taste reactivity behaviors in conscious rats. Chem. Senses, 38, 705-717.

Rizzone, M., Lanotte, M., Bergamasco, B., Tavella, A., Torre, E., Faccani, G., Melcarne, A. \& Lopiano, L. (2001) Deep brain stimulation of the subthalamic nucleus in Parkinson's disease: effects of variation in stimulation parameters. J. Neurol. Neurosur. Ps., 71, 215-219.

Robinson, M.J., Warlow, S.M. \& Berridge, K.C. (2014) Optogenetic excitation of central amygdala amplifies and narrows incentive motivation to pursue one reward above another. J. Neurosci., 34, 16567-16580. 
Rosenkranz, J.A. \& Grace, A.A. (1999) Modulation of basolateral amygdala neuronal firing and afferent drive by dopamine receptor activation in vivo. J. Neurosci., 19, 11027-11039.

Rouaud, T., Lardeux, S., Panayotis, N., Paleressompoulle, D., Cador, M. \& Baunez, C. (2010) Reducing the desire for cocaine with subthalamic nucleus deep brain stimulation. Proc. Natl. Acad. Sci. USA, 107, 1196-1200.

Sah, P., Faber, E.S., Lopez De Armentia, M. \& Power, J. (2003) The amygdaloid complex: anatomy and physiology. Physiol. Rev., 83, 803-834.

Sani, S., Jobe, K., Smith, A., Kordower, J.H. \& Bakay, R.A.E. (2007) Deep brain stimulation for treatment of obesity in rats. J. Neurosurg., 107, 809813.

Saper, C.B., Chou, T.C. \& Elmquist, J.K. (2002) The need to feed: homeostatic and hedonic control of eating. Neuron, 36, 199-211.

Schwalb, J.M. \& Hamani, C. (2008) The history and future of deep brain stimulation. Neurotherapeutics, 5, 3-13.

Seeley, R.J., Galaverna, O., Schulkin, J., Epstein, A.N. \& Grill, H.J. (1993) Lesions of the central nucleus of the amygdala. II: effects on intraoral $\mathrm{NaCl}$ intake. Behav. Brain Res., 59, 19-25.

Spybrook, J., Bloom, H., Congdon, R., Hill, C., Martínez, A. \& Raudenbush, S.W. (2011) Optimal design for longitudinal and multilevel research: Documentation for the "Optimal design" software version 3.0. 2015, 215.

Sternson, S.M. (2013) Hypothalamic survival circuits: blueprints for purposive behaviors. Neuron, 77, 810-824.

Swanson, L.W. \& Petrovich, G.D. (1998) What is the amygdala? Trends Neurosci., 21, 323-331.

Tindell, A.J., Berridge, K.C. \& Aldridge, J.W. (2004) Ventral pallidal representation of pavlovian cues and reward: population and rate codes. J. Neurosci., 24, 1058-1069.
Tindell, A.J., Smith, K.S., Pecina, S., Berridge, K.C. \& Aldridge, J.W. (2006) Ventral pallidum firing codes hedonic reward: when a bad taste turns good. J. Neurophysiol., 96, 2399-2409.

Touzani, K., Taghzouti, K. \& Velley, L. (1997) Increase of the aversive value of taste stimuli following ibotenic acid lesion of the central amygdaloid nucleus in the rat. Behav. Brain Res., 88, 133-142.

Tye, K.M., Prakash, R., Kim, S.Y., Fenno, L.E., Grosenick, L., Zarabi, H., Thompson, K.R., Gradinaru, V. et al. (2011) Amygdala circuitry mediating reversible and bidirectional control of anxiety. Nature, 471, $358-362$.

Wagenaar, D.A. \& Potter, S.M. (2002) Real-time multi-channel stimulus artifact suppression by local curve fitting. J. Neurosci. Meth., 120, 113 120.

Weaver, F.M., Follett, K., Stern, M., Hur, K., Harris, C., Marks, W.J. Jr, Rothlind, J., Sagher, O. et al. (2009) Bilateral deep brain stimulation vs best medical therapy for patients with advanced Parkinson disease A randomized controlled trial. JAMA-J. Am. Med. Assoc., 301, 63-73.

Will, M., Franzblau, E. \& Kelley, A. (2004) The amygdala is critical for opioid-mediated binge eating of fat. NeuroReport, 15, 1857-1860.

Will, M.J., Pritchett, C.E., Parker, K.E., Sawani, A.M., Ma, H. \& Lai, A.Y. (2009) Behavioral characterization of amygdala involvement in mediating intra-accumbens opioid-driven feeding behavior. Behav. Neurosci., 123, 781-793.

Wu, Y.R., Levy, R., Ashby, P., Tasker, R.R. \& Dostrovsky, J.O. (2001) Does stimulation of the GPi control dyskinesia by activating inhibitory axons? Movement Disord., 16, 208-216.

Wurtz, R.H. \& Olds, J. (1963) Amygdaloid stimulation and operant reinforcement in the rat. J. Comp. Physiol. Psych., 56, 941-949. 